\title{
Synthesis of 3-Aminopyrrolidin-2-ones by an Intramolecular reaction of Aziridinecarboxamides.
}

\author{
Ikuo Funaki, Lambertus Thijs and Binne Zwanenburg* \\ Department of Organic Chemistry, NSR Center for Molecular Structure, Design and Synthesis, University of Nijmegen, \\ Toernooiveld, 6525 ED Nijmegen, The Netherlands
}

\begin{abstract}
The synthesis of 4,5-disubstituted-3-amino- $\boldsymbol{\gamma}$-lactams from $\mathrm{N}$-substituted aziridinecarboxamides is described. Bicyclic aziridine derivatives were obtained from $1-\mathrm{N}-\mathrm{Boc}$-aziridinecarboxamides under kinetically controlled conditions. Oxiranecarboxamides showed similiar behavior and gave 3-hydroxylactams. Copyright $\odot 1996$ Elsevier Science Ltd
\end{abstract}

\section{INTRODUCTION}

Conformationally restricted dipeptide mimics have received extensive attention for the study of the relationships between biological activity, selectivity and peptide structures. ${ }^{1}$ The utilization of 3 -amino- $\gamma-, \delta$-and $\varepsilon$-lactams is of special interest in this context. ${ }^{1 p}, 2 \mathrm{a}$ Lactams are considered to provide a rigid structural unit in the backbone system of the mimics. Several research groups have designed lactam-bridged dipeptides e.g. 1 as conformationally restricted inhibitor mimics. 2,3

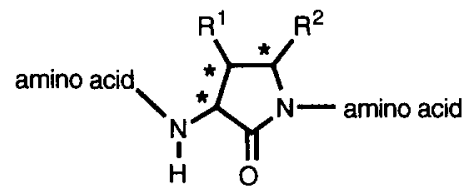

1

Most of the substituted $\gamma$-lactam rings reported were prepared in a stereospecific manner from the corresponding amino acids. These strategies often suffer from racemization at the stereogenic centers, or only allow a limited variation of substituents. To investigate structure-activity relationships, synthetic methods producing lactams from non-natural chiral sources are desired, because those methods would allow a more precise design of mimics. In this paper, the synthesis of 4,5-disubstituted 3-amino- $\gamma$-lactams 2 is reported, using intramolecular aziridine ring-opening reactions, whereby the chirality is transferred from the aziridine ring to the ring-expanded heterocycle (Scheme 1).

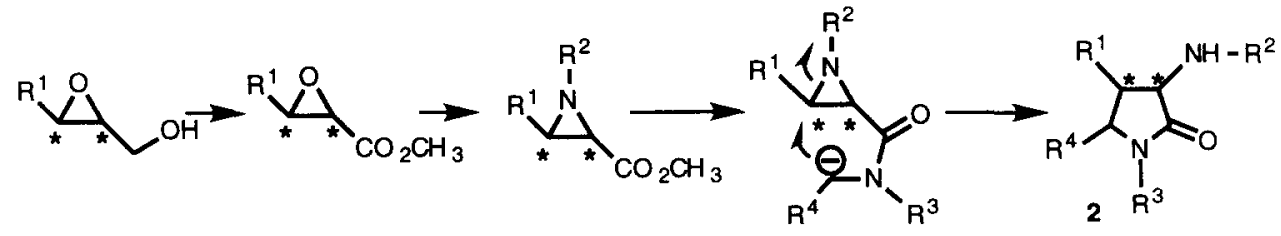

Scheme 1 
The required aziridines are available by the methods used and (partly) developed in the Nijmegen laboratory. ${ }^{4}$ Since enantiopure aziridinecarboxamides 5 can be prepared readily from enantiopure oxiranecarboxylic esters whose synthesis has been well established, 4,5 the strategy shown in Scheme 1 allows the preparation of various highly-substituted $\gamma$-lactams. In addition, it should be noted that intramolecular ringopening reactions of aziridines have been scarcely studied $6,7,8$ in contrast to intermolecular reactions. 9,10 It is also of interest to compare intramolecular ring-opening reactions of aziridines with those of the corresponding epoxides.

\section{RESULTS and DISCUSSION}

Activated aziridinecarboxamides $\mathbf{4}$ and 5 were prepared from aziridinecarboxylic esters $\mathbf{3}$ as shown in Scheme 2. Literature reports suggest that strong activation of the aziridine-ring system may be necessary to accomplish the desired ring-opening reactions. ${ }^{4,9}$ Therefore, 4-tolylsulfonyl (Ts) and tert-butoxycarbonyl (Boc) groups were introduced onto the aziridine nitrogen atom. Diethyl aminomalonate was chosen as the amine moiety of the carboxamides because the 'soft' nucleophilic character of malonyl carbanions was expected to be appropriate for the rather 'soft' aziridine ring carbons. After either the Ts or the Boc group was introduced, the activated aziridines were hydrolysed with $\mathrm{LiOH} / \mathrm{H}_{2} \mathrm{O}$, followed by DCC/HOBT condensation to afford aziridinecarboxamides $\mathbf{4}$ and $\mathbf{5}$ in high overall yields.

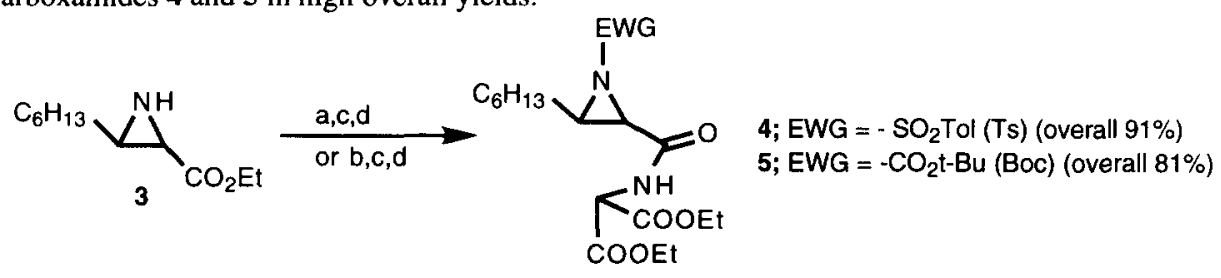

Reagents and Conditions: (a) (t-Boc) ${ }_{2} \mathrm{O}, \mathrm{DMAP}, \mathrm{CH}_{2} \mathrm{Cl}_{2}$; (b) TsCl, $\mathrm{Et}_{3} \mathrm{~N}, \mathrm{DMAP}, \mathrm{CH}_{2} \mathrm{Cl}_{2}$; (c) $\mathrm{LiOH}$, $\mathrm{H}_{2} \mathrm{O}$, THF; (d) DCC, diethyl aminomalonate, 1-hydroxybenzotriazole (HOBT), EtOAC.

Scheme 2

Treatment of (rac., trans) tosyl-activated aziridinecarboxamide 4 with a catalytic amount of sodium ethoxide in absolute ethanol at room temperature produced pyrrolidinone $\mathbf{6}$ in high yield (Scheme 3 ). The same product was isolated when substrate 4 was treated with LDA in THF or pyridine in chloroform, albeit in much lower yields.

The product 6 was proven to have a five-membered ring system by NMR proton-decoupling analysis. It was impossible, however, to determine the relative configurations at $\mathrm{C}-3$ and $\mathrm{C}-4$ from the coupling constants. Therefore an X-ray crystallographic analysis was performed which showed unambiguously the relative stereochemistry in 6 to be trans as shown in Figure 1.11 The formation of this five-membered heterocycle 6 clearly takes place by an intramolecular substitution reaction with inversion of configuration at the C-3 of the starting aziridine.

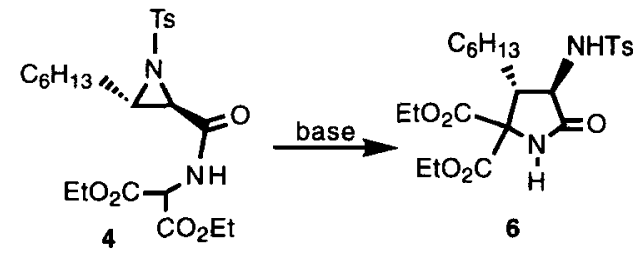

\begin{tabular}{llc} 
Entry & Reaction conditions & Yield (\%) \\
\hline 1 & $\begin{array}{l}\text { cat. NaOEt/EtOH } \\
\text { nt, overnight }\end{array}$ & 88 \\
2 & $\begin{array}{l}1.1 \text { eq. LDATHF } \\
-78{ }^{\circ} \mathrm{C} \text { to } \mathrm{rt} \text {, overnight } \\
3\end{array}$ & $\begin{array}{l}\text { excess pyridine/ } \mathrm{CHCl} \\
\text { nt, overnight }\end{array}$ \\
\hline
\end{tabular}

Scheme 3 


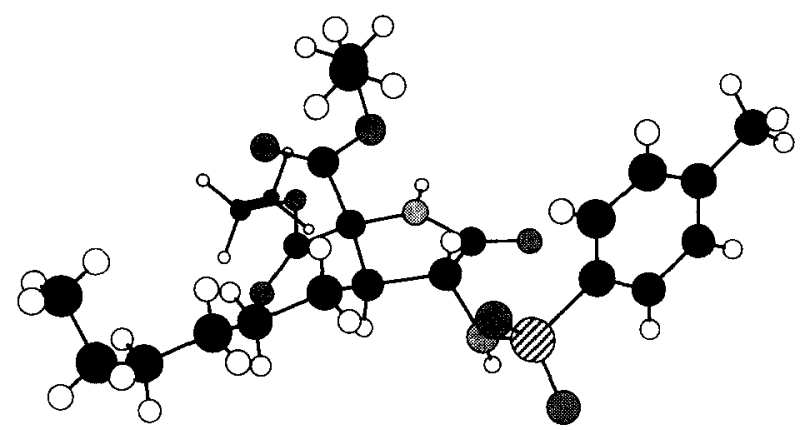

Figure 1. X-ray diffraction analysis of pyrrolidinone 6 .

The optimum conditions for intramolecular ring opening (i.e., catalytic NaOEt/EtOH) were also applied to the Boc-activated aziridinecarboxamide 5. This reaction resulted in the formation of pyrrolidinone 7 in $78 \%$ yield as a single isomer (Scheme 4). NMR analysis showed that the product $\mathbf{7}$ is a five-membered heterocycle.

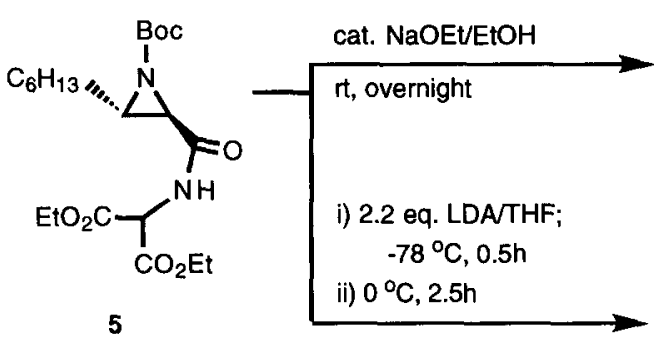

Scheme 4<smiles>CCOC(=O)C1(C(=O)OCC)NC(=O)[C@H](NC(=O)OCc2ccccc2)[C@H]1c1ccccc1</smiles><smiles>CCOCC(OCC)N1C(=O)C2C(C1=O)N2c1ccccc1</smiles>

$8(73 \%)$

In contrast, an unexpected result was obtained when substrate 5 was treated under kinetically controlled reaction conditions (LDA in THF at $0^{\circ} \mathrm{C}$ ), namely the formation of the bicyclic aziridine 8 in high yield $(73 \%)$. Interestingly, the aziridine moiety is retained in this product and an acyl substitution at the Boc has taken place. Some more examples of this type of bicyclic aziridines are collated in Table 1.

The thermodynamically controlled reaction conditions ( $\mathrm{NaOEt}$ in $\mathrm{EtOH}$ at room temperature) of these intramolecular reactions caused abstraction of the malonyl proton, leading to attack at $\mathrm{C}-3$ of the aziridine ring (Scheme 4). Under kinetically controlled reaction conditions (LDA in THF at $0^{\circ} \mathrm{C}$ ), however, LDA abstracted the proton attached to the amide-nitrogen, and the anion generated thus is 'hard' enough not to attack at the 'soft' aziridine-ring carbons but at the 'hard' carbonyl carbon. ${ }^{12}$ In all of the entries given in Table 1, aziridine ringopening reactions did not occur since the nitrogen-anion did not attack the aziridine ring carbons.

The synthesis of 3,4-cis-pyrrolidinone was then investigated in order to confirm the stereochemistry of the pyrrolidinone 7 since the measurement of ${ }^{1} \mathrm{H}-\mathrm{NMR}$ coupling constants was not possible. The cisaziridinecarboxamide $\mathbf{1 7}$ derived from the cis-aziridinecarboxylate disappointingly failed to react under the standard conditions of this method (Scheme 5). It is assumed that the hexyl group on aziridine C-3 interferes substantially with the bulky malonyl group; and the same interference could explain the absence of C-2 ringcarbon attack leading to an azetidinone. ${ }^{13 a}$ In comparable oxiranecarboxamide systems intramolecular attack has been observed at the $\mathrm{C}-2$ position, albeit only with a methyl as alkyl substituent. ${ }^{13 b-\mathrm{f}}$ 
Table 1

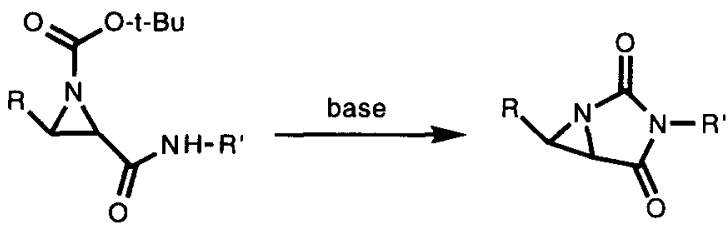

\begin{tabular}{|c|c|c|c|c|c|c|}
\hline Entry & Substrate & A & $\mathbf{R}^{\prime}$ & Reaction Conditions & Yield (\%) & Product \\
\hline 1 & 5 & Hex & $\mathrm{CH}\left(\mathrm{CO}_{2} \mathrm{Et}\right)_{2}$ & $\begin{array}{l}2.2 \text { eq. LDATHF; } \\
-78^{\circ} \mathrm{C}, 0.5 \mathrm{~h}, 0^{\circ} \mathrm{C}, 2.5 \mathrm{~h}\end{array}$ & 73 & 8 \\
\hline 2 & 9 & $\mathrm{Bu}$ & $\mathrm{CH}_{2} \mathrm{C}(\mathrm{O}) \mathrm{Ph}$ & $\begin{array}{l}\text { cat. t-BuOKJTHF; } \\
\text { rt, } 6 \mathrm{~h}\end{array}$ & 9 & 13 \\
\hline 3 & 10 & Hex & $\mathrm{CH}_{2} \mathrm{CO}_{2} \mathrm{Me}$ & $\begin{array}{l}\text { cat. NaOMe/MeOH; } \\
\text { rt, overnight }\end{array}$ & 52 & 14 \\
\hline 4 & 11 & Hex & $\mathrm{CH}_{2} \mathrm{Ph}$ & $\begin{array}{l}\text { cat. t-BuOK TTHF; } \\
\text { tt, overnight }\end{array}$ & 65 & 15 \\
\hline 5 & 12 & $\mathrm{PhCH}_{2}$ & $\mathrm{CH}_{2} \mathrm{C}(\mathrm{O}) \mathrm{Ph}$ & $\begin{array}{l}\text { cat. t-BuOK/THF; } \\
0^{\circ} \mathrm{C}, 3.5 \mathrm{~h}, \mathrm{rt}, 2.5 \mathrm{~h}\end{array}$ & 87 & 16 \\
\hline
\end{tabular}

Another approach to a cis sustituted pyrrolidinone was more successful. Intermolecular ring-opening reactions of aziridines have been investigated extensively 9,10 Hydriodic acid is known to react with activated aziridinecarboxamides, ${ }^{9 e}$ where iodide attacks selectively at C-3. Following the introduction of iodine in an $\mathrm{S}_{\mathrm{N}} 2$ fashion, it may be substituted through subsequent attack of malonyl carbanions. When $N$-Bocaziridinecarboxamide 5 was treated with hydriodic acid in acetone for $3 \mathrm{~h}$ at $0^{\circ} \mathrm{C}$, followed by the ring-closure conditions (1.5 eq. $\mathrm{NaOEt} / \mathrm{EtOH}$ ), iodide opened the aziridine ring and then participated as a leaving group (Scheme 6). The reaction presumably proceeded through double inversion leading to the 3,4-cis-pyrrolidinone 18, together with the 3,4-trans-pyrrolidinone 7 (15\% yield). The trans-pyrrolidinone 7 may have been produced through the direct intramolecular reaction of unreacted starting material 5 (according to Scheme 4) without the assistance of iodide.

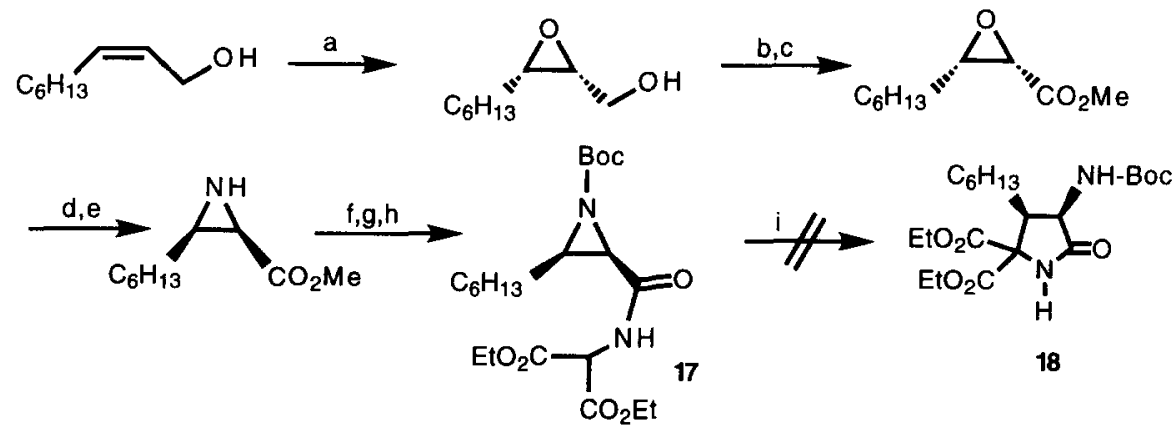

Reactions and Reagents: (a) Sharpless epoxidation; (b) Ru cat., $\mathrm{NalO}_{4}$; (c) Diazomethane; (d) $\mathrm{NaN}_{3}$. $\mathrm{NH}_{4} \mathrm{Cl} / \mathrm{EtOH}$; (e) $\mathrm{PPh}_{3}$; (f) di-t-Butoxy-dicarbonate, DMAP; (g) aq. LiOH/THF; (h) Diethyl aminomalonate, DCC/ EtOAC; (i) cat. NaOEt/EtOH.

\section{Scheme 5}

The pyrrolidinones 7 and 18 were subjected to 2D-NOESY NMR analysis in order to confirm the configuration of 3,4-trans- and cis- $\mathrm{N}$-Boc-pyrrolidinones. ${ }^{14} \mathrm{C} 4-\mathrm{H}$ of 7 showed interaction with the $\mathrm{N}-\mathrm{H}$ of the carbamoyl group. Contrastingly the $\mathrm{C}_{4}-\mathrm{H}$ of 18 did not interact with the $\mathrm{N}-\mathrm{H}$ of the carbamoyl function but 
instead interacted with $\mathrm{C}_{3}-\mathrm{H}$. These observations support the assigned structure, namely, the pyrrolidinone 7 has a 3,4-trans configuration, whilst in the pyrrolidinone 18 this is cis.

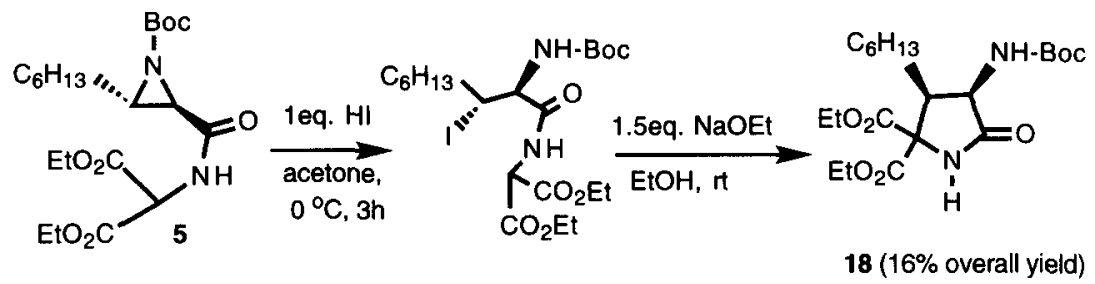

Scheme 6

The new methodology shown in Schemes 3 and 4 was also applied to the synthesis of 3hydroxypyrrolidinone. As anticipated from the reported result of a similar epoxide ring-opening reaction, ${ }^{15}$ intramolecular reaction of the oxiranecarboxamide analogue 19 took place in the same manner as for aziridinecarboxamides. When the epoxide analogue 19 was treated with a catalytic amount of sodium ethoxide in ethanol, pyrrolidinone $\mathbf{2 0}$ was obtained (Scheme 7).

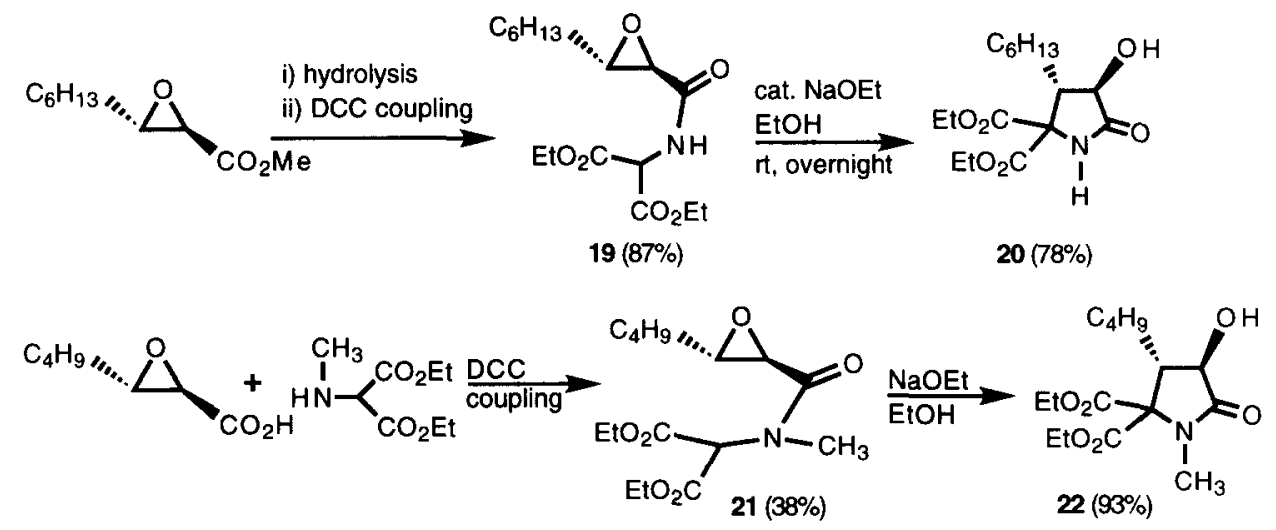

Scheme 7

Substitution of nitrogen in the oxiranecarboxamide with a methyl group as in $\mathbf{2 1}$ did not change the outcome of the reaction, again a pyrrolidinone e.g. 22 was obtained. Although the aziridine analogue of 21 could not be prepared due to the difficulty of the amide-bond forming reaction, the result of $\mathbf{2 1}$ is indicative of the reaction of the corresponding aziridinecarboxamide, since the reaction characteristics are virtually the same.

The aziridinecarboxamides $\mathbf{4}$ and $\mathbf{5}$ carry two ester units in the malonyl moiety. The synthetic scope of the intramolecular ring opening would improve if such a reaction could also be performed with an aziridinecarboxamide containing only one ester function. Such a substrate, i.e. 23 could be prepared by a DCC coupling of an aziridinecarboxylic acid and $N$-benzylglycine methyl ester. Treatment of substrate 23 with potassium t-butoxide in THF resulted in the formation of azetidinone 24, albeit in low yield (19\%), together with unreacted 23 (Scheme 8). With LDA the yield was even lower (7\%). No five-membered ring product could be detected, although for the tosyl-activated aziridine ring an intramolecular reaction at C-3 would have been expected. ${ }^{16}$ Other bases, such as Triton B and sodium bis(trimethylsilyl)amide yielded no cyclization products. Interestingly, azetidinone $\mathbf{2 4}$ has the cis configuration as was deduced from the coupling constant for $\mathrm{H}-3$ and $\mathrm{H}$ 4 in the ${ }^{1} \mathrm{H}-\mathrm{NMR}$ spectrum. A satisfactory explanation for this stereochemical outcome cannot be given yet. 


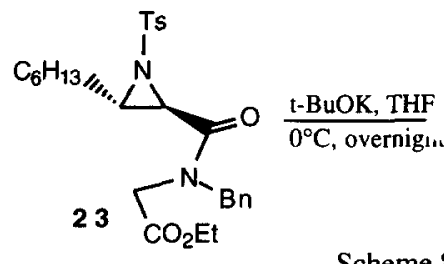

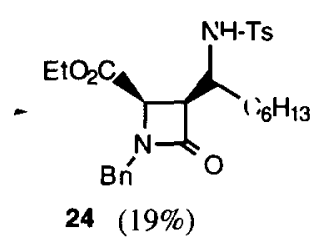

Scheme 8

The aziridinecarboxamide 25 corresponding to 23 but without the $N$-benzyl group, was also prepared. However, base treatment only led to decomposition products.

The failure of these cyclization reactions prompted us to try alternative methods, such as a decarboxylation reaction.

Although pyrrolidinone 7 could not be converted into the corresponding monoethoxycarbonyl pyrrolidinone 26 by treatment with $\mathrm{NaCl}-\mathrm{H}_{2} \mathrm{O}-\mathrm{DMSO},{ }^{17}$ it was obtained by employing $\mathrm{LiBr}-\mathrm{H}_{2} \mathrm{O}-1,3-$ dimethyl-2imidazolidinone (DMI; $\left.155^{\circ} \mathrm{C}, 4 \mathrm{~h}\right)^{18}$ (Scheme 14). The product was a 1:1 inseparable mixture of $\mathrm{C}-5$ isomers which could be analyzed by ${ }^{1} \mathrm{H}$-NMR spectroscopy. When the same procedure was applied to the pyrrolidinone 6, mono-de-ethoxycarbonylation was also achieved and the two C-5 isomers $27 \mathbf{a} / 27 \mathbf{b}$ formed were readily separated by column chromatography. Unfortunately, an attempt to determine the stereochemistry of the two isomers $27 \mathbf{a} / \mathbf{2 7}$ b by $2 \mathrm{D}-\mathrm{NOESY}{ }^{1} \mathrm{H}$ NMR analysis failed to provide a clear answer.

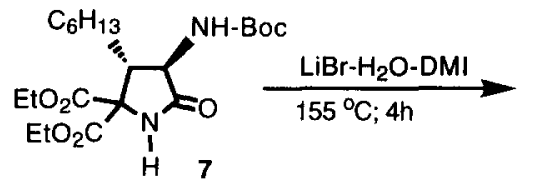<smiles>CCOC(=O)N[C@@H]1[C@H](NC(=O)OCc2ccccc2)C(=O)N[C@@H]1C(=O)OCC</smiles>

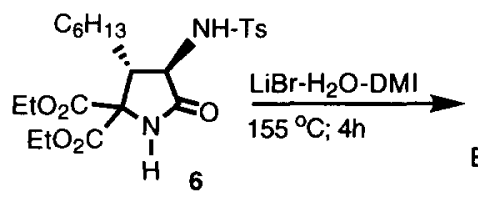

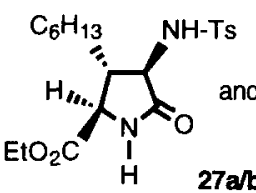

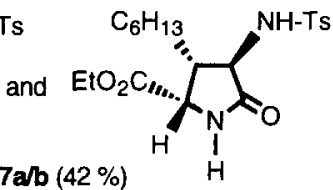

Scheme 9

\section{CONCLUSION}

In conclusion, a novel synthetic strategy for the preparation of 3-amino-4-hexyl-5-ethoxycarbonylpyrrolidin-2-ones is described. The intramolecular ring-opening reaction of activated aziridines proceeds smoothly under mild reaction conditions. During the course of the reactions, the relative configuration of aziridines was transferred successfully to the respective pyrrolidinones. The configurations of the pyrrolidinones were confirmed by X-ray analysis and 2D NOESY NMR studies. The same type of intramolecular ring-opening reaction also took place with epoxide analogues. In the case of $N$-Boc aziridines, bicyclic aziridines were obtained by altering the reaction conditions. The 4,5-cis-pyrrolidinone could be synthesized from the same starting material 5 by a twostep reaction using hydriodic acid and subsequent treatment with $\mathrm{NaOEt}$.

A variety of ethyl or methyl 3-alkylaziridine-2-carboxylates are readily available as starting materials by reported methods implying that this methodology has, in principle, a wide scope. ${ }^{4}$ 


\section{EXPERIMENTAL SECTION}

Proton magnetic resonance spectra were measured on a Bruker WH-90, Bruker AC-100 or a Bruker AM400 spectrometer. Chemical shift values are reported as $\delta$-values relative to tetramethylsilane as an internal standard. Mass spectra were obtained with a double focussing VG 7070E spectrometer. IR spectra were recorded on a Perkin-Elmer 298 infrared spectrophotometer. Melting points were measured with a Reichert Thermopan microscope and are uncorrected. Recrystallizations were carried out using hexane-EtOAc unless stated otherwise. GLC was conducted with a Hewlett-Packard HP 5890 gas chromatograph, using a capillary column $(25 \mathrm{~m})$ of $\mathrm{HP}-1$, and nitrogen at $2 \mathrm{ml} / \mathrm{min}(0.5 \mathrm{~atm})$ as the carrier gas. Commercial $\mathrm{n}$-BuLi solution in hexane (ca. $1.6 \mathrm{M})$ was purchased from Merck. Hexane was distilled from calcium hydride. Diethyl ether $\left(\mathrm{Et}_{2} \mathrm{O}\right)$ was pre-dried over calcium chloride, then distilled from calcium hydride and again from sodium hydride. Tetrahydrofuran was freshly distilled from lithium aluminum hydride. 1,2-Dimethoxyethane was distilled from sodium hydride. Acetonitrile was distilled from phosphorus pentoxide. N,N,-Dimethylformamide (DMF) was purified first by azeotropic distillation with benzene, and, after treatment with barium oxide, it was distilled in vacuo under nitrogen. All other solvents were obtained commercially and used without further purification. Thin-layer chromatography (TLC) was performed on silica gel F-254 plates (thickness $0.25 \mathrm{~mm}$ ). Spots were visualized with a UV hand lamp, $\mathrm{K}_{2} \mathrm{Cr}_{2} \mathrm{O}_{7}$ solution in dil. $\mathrm{H}_{2} \mathrm{SO}_{4}$ or $\mathrm{Cl}_{2}-\mathrm{TDM} .{ }^{19}$ Column chromatography was carried out using silica $60 \mathrm{H}$ (for flash chromatography, Merck, art. nr. 7736) or silica 60 (Merck, art. nr. 7734).

Standard procedure for preparation of aziridinecarboxamides: trans-1-(tertButoxycarbonyl)-3-hexylaziridine-2-carbonylaminomalonic acid diethylester (5). All the 1-(tertbutoxycarbonyl)-3-hexylaziridine-2-carboxamides were prepared from the corresponding 3-hexylaziridine-2carboxylic acid methyl (or ethyl) esters 3 according to this procedure: To a solution of $3(1.0 \mathrm{~g} ; 5.4 \mathrm{mmol})$ in dry $\mathrm{CH}_{2} \mathrm{Cl}_{2}(6 \mathrm{~mL})$ under argon were added 4-(dimethylamino)pyridine $(1.6 \mathrm{~g} ; 14 \mathrm{mmol})$ and di-tert-butyl dicarbonate $(2.9 \mathrm{~g}, 14 \mathrm{mmol})$. The solution was stirred at room temperature for $2 \mathrm{~h}$, quenched with saturated aqueous $\mathrm{NH}_{4} \mathrm{Cl}$ solution $(25 \mathrm{~mL})$, poured into water, and extracted with ether $(3 \times 50 \mathrm{~mL})$. The combined extracts were washed with saturated aqueous $\mathrm{KHSO}_{4}$ solution, saturated aqueous $\mathrm{Na}_{2} \mathrm{CO}_{3}$ solution and brine, dried $\left(\mathrm{MgSO}_{4}\right)$, filtered, and concentrated in vacuo. Chromatography (silica, hexane-EtOAc (7:1)) of the residue gave the $N$-Boc derivative $(1.5 \mathrm{~g}, 97 \%)$. The pure product $(0.77 \mathrm{~g}, 2.7 \mathrm{mmol})$ was dissolved in THF $(14 \mathrm{~mL})$ and treated with a $1.0 \mathrm{~N}$ aqueous solution of lithium hydroxide $(5.4 \mathrm{~mL})$. The mixture was stirred overnight at room temperature and then acidified with dilute $\mathrm{H}_{2} \mathrm{SO}_{4}$ to $\mathrm{pH} 3$ and extracted with $\mathrm{Et}_{2} \mathrm{O}(3 \times 30 \mathrm{~mL})$. The combined organic extracts were dried $\left(\mathrm{MgSO}_{4}\right)$, filtered and concentrated in vacuo to give $N$-(tertbutoxycarbonyl)-3-hexylaziridine-2-carboxylic acid $(0.72 \mathrm{~g}, 98 \%)$ as a colorless oil. The acid was converted into 5 without further purification by using dicyclohexylcarbodiimide (DCC) as a condensing agent. The acid $(0.69 \mathrm{~g}$, $2.5 \mathrm{mmol})$, diethyl aminomalonate ${ }^{20}(0.44 \mathrm{~g}, 2.5 \mathrm{mmol})$ and 1-hydroxybenzotriazole $(0.38 \mathrm{~g}, 2.5 \mathrm{mmol})$ were dissolved in EtOAc $(25 \mathrm{~mL})$. The solution was stirred and cooled in an ice bath and then treated with DCC $(0.57$ $\mathrm{g}, 2.8 \mathrm{mmol}$ ). The mixture was stirred for one hour at $0^{\circ} \mathrm{C}$ and then overnight at room temperature. $N, N^{\prime}$ Dicyclohexylurea which separated was removed by filtration. The organic filtrate was washed with a $10 \%$ solution of citric acid in water $(100 \mathrm{~mL})$ and brine, dried $\left(\mathrm{MgSO}_{4}\right)$, filtered, and concentrated in vacuo. Chromatography (silica, hexane-EtOAc (5:1)) gave $5(0.91 \mathrm{~g}, 85 \%)$.

Ethyl trans-1-(tert-butoxycarbonyl)-3-hexylaziridine-2-carboxylate. Oil; IR ( $\left.\mathrm{CCl}_{4}\right) 2920$, $1740,1530,1310,1150 \mathrm{~cm}^{-1}$; ${ }^{\mathrm{H}} \mathrm{NMR}\left(\mathrm{CDCl}_{3}\right) \delta 0.88$ (br. t, $\left.3 \mathrm{H}, \mathrm{CH}_{3}\left(\mathrm{CH}_{2}\right)_{5}\right), 1.23-1.46(\mathrm{~m}, 22 \mathrm{H}$, $\left.\mathrm{CH}_{3}\left(\mathrm{CH}_{2}\right)_{5}, \mathrm{OCH}_{2} \mathrm{CH}_{3}, \mathrm{C}\left(\mathrm{CH}_{3}\right)_{3}\right), 2.7-2.82(\mathrm{~m}, 2 \mathrm{H}, \mathrm{CHNCH}), 4.21+4.23(2 \times q, \mathrm{~J}=7.2 \mathrm{~Hz}, 2 \mathrm{H}$, $\left.\mathrm{OCH}_{2} \mathrm{CH}_{3}\right)$. HRMS $(\mathrm{M}+1)^{+}$; Calcd for $\mathrm{C}_{16} \mathrm{H}_{30} \mathrm{NO}_{4}: 300.2175$; Found: 300.21761 . 
trans-1-(tert-Butoxycarbonyl)-3-hexylaziridine-2-carbonylaminomalonic acid diethylester (5). Oil; IR $\left(\mathrm{CCl}_{4}\right) 3400,2930,1760,1730,1690,1500 \mathrm{~cm}^{-1} ;{ }^{1} \mathrm{H}$ NMR $\left(\mathrm{CDCl}_{3}\right) \delta 0.88$ (br t, 3H, $\left.\left.\mathrm{CH}_{3}\left(\mathrm{CH}_{2}\right)_{5}\right), 1.22-1.47\left(\mathrm{~m}, 25 \mathrm{H}, \mathrm{CH}_{3}\left(\mathrm{CH}_{2}\right)_{5}, 2 \mathrm{xOCH}_{2} \mathrm{CH}_{3}, \mathrm{C}\left(\mathrm{CH}_{3}\right)_{3}\right)\right), 2.5-2.7(\mathrm{~m}, 1 \mathrm{H}, \mathrm{Hex}-\mathrm{CHN}), 2.83$ $(\mathrm{d}, \mathrm{J}=2.9 \mathrm{~Hz}, 1 \mathrm{H}, \mathrm{NCHCO}), 4.24+4.26\left(2 \mathrm{xq}, \mathrm{J}=7.1 \mathrm{~Hz}, 4 \mathrm{H}, 2 \mathrm{xOCH}_{2} \mathrm{CH}_{3}\right), 5.15(\mathrm{~d}, \mathrm{~J}=7.4 \mathrm{~Hz}, 1 \mathrm{H}$, $\mathrm{NHCH}), 7.06(\mathrm{~d}, \mathrm{~J}=7.4 \mathrm{~Hz}, 1 \mathrm{H}, \mathrm{NH})$. HRMS $(\mathrm{M})^{+}$; Calcd for $\mathrm{C}_{21} \mathrm{H}_{36} \mathrm{~N}_{2} \mathrm{O}_{7}: 428.2522$; Found: 428.25273.

Ethyl trans-3-hexyl-1-(toluene-4-sulfonyl)aziridine-2-carboxylate. To a solution of 3 (1.3 g, $6.5 \mathrm{mmol})$ dissolved in dry $\mathrm{CH}_{2} \mathrm{Cl}_{2}(33 \mathrm{~mL})$ and triethylamine $(1.8 \mathrm{~mL}, 13 \mathrm{mmol})$, a catalytic amount of 4 dimethylaminopyridine and tosyl chloride $(1.9 \mathrm{~g}, 9.8 \mathrm{mmol})$ were added sequentially under argon at $0{ }^{\circ} \mathrm{C}$. After $30 \mathrm{~min}$, the mixture was allowed to warm to room temperature, and then stirred for $2 \mathrm{~h}$ at this temperature. The mixture was poured into a mixture of $\mathrm{Et}_{2} \mathrm{O}(50 \mathrm{~mL})$ and brine $(100 \mathrm{~mL})$, and extracted with $\mathrm{Et}_{2} \mathrm{O}(3 \times 40 \mathrm{~mL})$. The combined organic extracts were washed with brine, dried $\left(\mathrm{MgSO}_{4}\right)$, filtered, and concentrated in vacuo. The residue was purified by column chromatography (silica, hexane-EtOAc (10:1)) to give ethyl $N$-tosyl aziridinecarboxylate $(2.1 \mathrm{~g}, 92 \%)$. mp. $63.5-64{ }^{\circ} \mathrm{C}$; IR $\left(\mathrm{CCl}_{4}\right) 3060,3030,2955,2925,2855,1740,1595$, $1460,1445,1380,1370,1335,1305,1290,1185,1165,1090,1035,920,705,690,685,635 \mathrm{~cm}^{-1} ;{ }^{1} \mathrm{H}$ NMR $\left(\mathrm{CDCl}_{3}\right) \delta 0.9\left(\mathrm{brt}, 3 \mathrm{H}, \mathrm{CH}_{3}\left(\mathrm{CH}_{2}\right)_{5}\right), 1.1-1.7\left(\mathrm{t}+\mathrm{m}, 11 \mathrm{H}, \mathrm{CH}_{3}\left(\mathrm{CH}_{2}\right)_{4} \mathrm{CH}_{2}, \mathrm{OCH}_{2} \mathrm{CH}_{3}\right), 1.95(\mathrm{~m}, 2 \mathrm{H}$, $\left.\mathrm{CH}_{3}\left(\mathrm{CH}_{2}\right)_{4} \mathrm{CH}_{2}\right), 2.4\left(\mathrm{~s}, 3 \mathrm{H}, \mathrm{PhCH}_{3}\right), 3.05(\mathrm{~m}, 1 \mathrm{H}, \mathrm{Hex}-\mathrm{CHN}), 3.25(\mathrm{~d}, \mathrm{~J}=3.5 \mathrm{~Hz}, 1 \mathrm{H}, \mathrm{NCHCO}), 4.15$ (q, $2 \mathrm{H}, \mathrm{OCH}_{2} \mathrm{CH}_{3}$ ), 7.3 (d, J = 7.5 Hz, $\left.2 \mathrm{H}, 3,5-\mathrm{Ph}\right), 7.85$ (d, J = $\left.7.5 \mathrm{~Hz}, 2 \mathrm{H}, 2,6-\mathrm{Ph}\right) . \mathrm{MS}(\mathrm{Cl}): \mathrm{m} / \mathrm{e}$ (relative intensity) 354 (19, M+1), 308 (6), 280 (100), 256 (13), 224 (5), 198 (63), 183 (54), 172 (8), 155 (11), 137 (9), 124 (10), 109 (13), 91 (3), 55 (15), 43 (41). Anal. Calcd. for $\mathrm{C}_{18} \mathrm{H}_{27} \mathrm{NO}_{4} \mathrm{~S}$ (353.482): C, 61.16; H, 7.70; $\mathrm{N}$, 3.96; Found: $\mathrm{C}, 61.42 ; \mathrm{H}, 7.72 ; \mathrm{N}, 4.02$.

3-Hexyl-1-(toluene-4-sulfonyl)aziridine-2-carbonylaminomalonic acid diethyl ester (4). This amino-malonic acid diethylester was prepared from ethyl $N$-tosyl-aziridinecarboxylate according to the standard method for $N$-Boc-aziridinecarboxamide in $92 \%$ yield. oil; IR $\left(\mathrm{CCl}_{4}\right) 3400,2920,1755,1690,1500$,

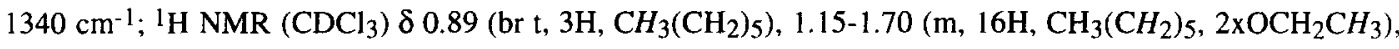
$2.44\left(\mathrm{~s}, 3 \mathrm{H}, \mathrm{PhCH}_{3}\right), 2.8-3.0(\mathrm{~m}, 1 \mathrm{H}, \mathrm{Hex}-\mathrm{CHN}), 3.35(\mathrm{~d}, \mathrm{~J}=4.0 \mathrm{~Hz}, 1 \mathrm{H}, \mathrm{NCHCO}), 4.17+4.20(2 \mathrm{xq}, \mathrm{J}=$ $\left.7.1 \mathrm{~Hz}, 4 \mathrm{H}, 2 \times \mathrm{OCH}_{2} \mathrm{CH}_{3}\right), 4.96(\mathrm{~d}, \mathrm{~J}=7.3 \mathrm{~Hz}, 1 \mathrm{H}, \mathrm{NHCH}), 6.8(\mathrm{~d}, \mathrm{~J}=7.5 \mathrm{~Hz}, 1 \mathrm{H}, \mathrm{NH}) .7 .35(\mathrm{~d}, \mathrm{~J}=8.4$ $\mathrm{Hz}, 2 \mathrm{H}, 3,5-\mathrm{Ph}), 7.88(\mathrm{~d}, \mathrm{~J}=8.3 \mathrm{~Hz}, 2 \mathrm{H}, 2,6-\mathrm{Ph})$. HRMS (M) ${ }^{+}$; Calcd for $\mathrm{C}_{23} \mathrm{H}_{34} \mathrm{~N}_{2} \mathrm{O}_{7} \mathrm{~S}: 482.2087$; Found: 482.20862 .

\section{Intramolecular ring opening reactions of aziridine (or oxirane)carboxamides :}

Method A; A representative procedure using NaOEt: $N$-Boc-aziridinecarboxamide 5 ( $0.77 \mathrm{~g}, 1.8 \mathrm{mmol})$ was dissolved in absolute $\mathrm{EtOH}(50 \mathrm{~mL})$, and a few drops of sodium ethoxide (1.3M in EtOH) were added under argon at room temperature. The reaction mixture was stirred overnight, then concentrated in vacuo. The residue was purified by column chromatography (silica, hexane-EtOAc (1:1)) to give pyrrolidinone $7(0.60 \mathrm{~g}, 78 \%)$.

Method $B ; A$ procedure using lithium diisopropylamide: $N$-Tosyl-aziridinecarboxamide $4(0.42 \mathrm{~g}, 0.87$ mmol) dissolved in dry THF ( $5 \mathrm{~mL}$ ) was added under argon to lithium diisopropylamide (LDA) solution ( $33 \mathrm{~mL}$, $0.029 \mathrm{M}$ in THF) cooled at $-78{ }^{\circ} \mathrm{C}$. The mixture was stirred at $-78^{\circ} \mathrm{C}$ for $30 \min$ at $0{ }^{\circ} \mathrm{C}$ for $2 \mathrm{~h}$, and then allowed to warm to room temperature and stirred overnight and then treated with saturated aqueous $\mathrm{NH}_{4} \mathrm{Cl}$ solution $(10 \mathrm{~mL})$, water, and extracted with EtOAc $(3 \times 40 \mathrm{~mL})$. The combined organic extracts were washed with brine, dried $\left(\mathrm{MgSO}_{4}\right)$, filtered and concentrated in vacuo. Column chromatography (silica,hexane-EtOAc (1:1)) gave pyrrolidinone $6(0.13 \mathrm{~g}, 31 \%)$.

Method $C ; A$ procedure using pyridine: Pyridine $(0.54 \mathrm{~mL}, 7.0 \mathrm{mmol})$ was added under argon to a chloroform solution $(0.35 \mathrm{~mL})$ of $4(0.25 \mathrm{~g}, 0.52 \mathrm{mmol})$ at room temperature. The mixture was stirred overnight and then treated with ice/water. The mixture was extracted with EtOAc $(3 \times 15 \mathrm{~mL})$, and the combined organic 
extracts were washed with dil. aqueous $\mathrm{CuSO}_{4}$ solution $(3 \times 25 \mathrm{~mL})$, brine, dried $\left(\mathrm{MgSO}_{4}\right)$, filtered and concentrated in vacuo. Column chromatography (silica, hexane-EtOAc (3:1) to (1:1)) of the residue gave $6(0.10$ $\mathrm{g}, 40 \%)$ and $4(0.08 \mathrm{~g}, 32 \%)$.

trans-3-(Toluene-4-sulfonyl)amino-4-hexyl-5,5-di(ethoxycarbonyl)pyrrolidin-2-one (6). mp. $78-80^{\circ} \mathrm{C}$; IR $\left(\mathrm{CCl}_{4}\right) 3420,3300,2920,1735,1540,1250 \mathrm{~cm}^{-1} ;{ }^{1} \mathrm{H} \mathrm{NMR}\left(\mathrm{CDCl}_{3}\right) \delta 0.90($ br t, J = 6.0 Hz. $\left.3 \mathrm{H}, \mathrm{CH}_{3}\left(\mathrm{CH}_{2}\right)_{5}\right), 1.1-1.7\left(\mathrm{~m}, 16 \mathrm{H}, \mathrm{CH}_{3}\left(\mathrm{CH}_{2}\right)_{5}, 2 \mathrm{XOCH}_{2} \mathrm{CH}_{3}\right), 2.3$ (s, 3H, PhCH 3$), 2.6-2.8(\mathrm{~m}, 1 \mathrm{H}, \mathrm{Hex}-$ $\mathrm{CH}), 4.0(\mathrm{dd}, \mathrm{J}=9.0,10 \mathrm{~Hz}, 1 \mathrm{H}, \mathrm{CHNH}), 4.29+4.31\left(2 \mathrm{xq}, \mathrm{J}=7 \mathrm{~Hz}, 4 \mathrm{H}, 2 \mathrm{xOCH}_{2} \mathrm{CH}_{3}\right), 5.7(\mathrm{br} \mathrm{d}, \mathrm{J}=9 \mathrm{~Hz}$, 1H, NH-Ts), 6.8 (br s, 1H, NHCO), 7.3 (d, J = $9 \mathrm{~Hz}, 2 \mathrm{H}, 3,5-\mathrm{Ph}$ ), 7.8 (d, J = $9 \mathrm{~Hz}, 2 \mathrm{H}, 2,6-\mathrm{Ph}$ ). Anal. Calcd. for $\mathrm{C}_{23} \mathrm{H}_{34} \mathrm{~N}_{2} \mathrm{O}_{7} \mathrm{~S}: \mathrm{C}, 57.24 ; \mathrm{H}, 7.10 ; \mathrm{N}, 5.80 ; \mathrm{S}, 6.64$; Found: C, 57.35; H, 7.07; N, 5.79; S, 6.75.

trans-3-(tert-Butoxycarbonyl)amino-4-hexyl-5,5-di(ethoxycarbonyl)pyrrolidin-2-one (7). mp. 144-145 ${ }^{\circ} \mathrm{C}$; IR $\left(\mathrm{CCl}_{4}\right) 3380,3200,2920,1720,1500,1370 \mathrm{~cm}^{-1} ;{ }^{1} \mathrm{H} \mathrm{NMR}\left(\mathrm{CDCl}_{3}\right) \delta 0.87(\mathrm{t}, \mathrm{J}=6.8 \mathrm{~Hz}$, $\left.3 \mathrm{H}, \mathrm{CH}_{3}\left(\mathrm{CH}_{2}\right)_{5}\right), 1.25-1.51\left(\mathrm{~m}, 24 \mathrm{H}, \mathrm{C}\left(\mathrm{CH}_{3}\right)_{3}, \mathrm{CH}_{3}\left(\mathrm{CH}_{2}\right)_{4} \mathrm{CHH}, 2 \mathrm{XOCH}_{2} \mathrm{CH}_{3}\right), 1.71-1.80(\mathrm{~m}, 1 \mathrm{H}$, $\left.\mathrm{CH}_{3}\left(\mathrm{CH}_{2}\right)_{4} \mathrm{CH} H\right), 2.6-2.8(\mathrm{~m}, 1 \mathrm{H}, \mathrm{Hex}-\mathrm{CH}), 4.23-4.32\left(\mathrm{~m}, 5 \mathrm{H}, \mathrm{CHNH}, 2 \mathrm{OCCH}_{2} \mathrm{CH}_{3}\right), 4.92(\mathrm{~d}, \mathrm{~J}=8.9 \mathrm{~Hz}$. $1 \mathrm{H}, \mathrm{NH}-\mathrm{CO}_{2} \mathrm{t}-\mathrm{Bu}$ ), 6.55 (br s $1 \mathrm{H}, \mathrm{NHCO}$ ). Anal.Calcd for $\mathrm{C}_{21} \mathrm{H}_{36} \mathrm{~N}_{2} \mathrm{O}_{7}$ : C, 58.86; $\mathrm{H}, 8.47 ; \mathrm{N}, 6.54$; Found: C,58.81; H, 8.42; N, 6.56 .

2-(6-Hexyl-2,4-dioxo-1,3-diaza-bicyclo[3,1,0]hex-3-yl)malonic acid diethyl ester (8). $N$ Boc-aziridinecarboxamide $5(0.50 \mathrm{~g}, 1.2 \mathrm{mmol})$ dissolved in dry THF ( $5 \mathrm{~mL})$ was added under argon to lithium diisopropylamide solution ( $30 \mathrm{~mL}, 0.088 \mathrm{M}$ in THF) cooled to $-78^{\circ} \mathrm{C}$. The mixture was stirred at $-78^{\circ} \mathrm{C}$ for 30 min and at $0{ }^{\circ} \mathrm{C}$ for $2.5 \mathrm{~h}$. The reaction mixture was then treated with saturated aqueous $\mathrm{NH}_{4} \mathrm{Cl}$ solution $(10 \mathrm{~mL})$, water, and extracted with EtOAc $(3 \times 40 \mathrm{~mL})$. The combined organic extracts were washed with brine, dried $\left(\mathrm{MgSO}_{4}\right)$, filtered and concentrated in vacuo. Column chromatography (silica, hexane-EtOAc (3:1)) of the residue gave bicyclic aziridine $8(0.31 \mathrm{~g}, 73 \%)$. oil; IR $\left(\mathrm{CCl}_{4}\right) 2920,1795,1740,1390,1250,1180 \mathrm{~cm}^{-1} ;{ }^{1} \mathrm{H} \mathrm{NMR}$ $\left(\mathrm{CDCl}_{3}\right) \delta$ 0.80-1.0 (br t, 3H, $\left.\mathrm{CH}_{3}\left(\mathrm{CH}_{2}\right)_{5}\right), 1.23-1.80\left(\mathrm{~m}, 16 \mathrm{H}, \mathrm{CH}_{3}\left(\mathrm{CH}_{2}\right)_{5}, 2 \mathrm{OCH}_{2} \mathrm{CH}_{3}\right), 2.8-2.9(\mathrm{~m}, 1 \mathrm{H}$, $\mathrm{Hex}-\mathrm{CH}), 3.22(\mathrm{~d}, \mathrm{~J}=3.1 \mathrm{~Hz}, 1 \mathrm{H}, \mathrm{NCHCO}), 4.29\left(\mathrm{q}, \mathrm{J}=6.9 \mathrm{~Hz}, 4 \mathrm{H}, 2 \mathrm{xOCH}_{2} \mathrm{CH}_{3}\right), 5.10(\mathrm{~s}, 1 \mathrm{H}$, $\left.\mathrm{CH}\left(\mathrm{CO}_{2} \mathrm{Et}\right)_{2}\right)$. HRMS (M)+; Calcd for $\mathrm{C}_{17} \mathrm{H}_{26} \mathrm{~N}_{2} \mathrm{O}_{6}$ : 354.1791; Found: 354.17886.

Ethyl trans-1-(tert-butoxycarbonyl)-3-butylaziridine-2-carboxylate. ${ }^{4} \mathrm{Oil}$; IR $\left(\mathrm{CCl}_{4}\right) 2960$, $1730,1520,1310,1225,1150 \mathrm{~cm}^{-1} ;{ }^{1} \mathrm{H}$ NMR $\left(\mathrm{CDCl}_{3}\right) \delta 0.89\left(\mathrm{br} \mathrm{t}, 3 \mathrm{H}, \mathrm{CH}_{3}\left(\mathrm{CH}_{2}\right)_{3}\right), 1.2-1.6(\mathrm{~m}, 18 \mathrm{H}$, $\left.\mathrm{CH}_{3}\left(\mathrm{CH}_{2}\right)_{3}, \mathrm{t}-\mathrm{Bu}, \mathrm{OCH}_{2} \mathrm{CH}_{3}\right), 2.7-2.85(\mathrm{~m}, 2 \mathrm{H}, \mathrm{CHNCH}), 4.21+4.24\left(2 \mathrm{xq}, \mathrm{J}=7.2 \mathrm{~Hz}, 2 \mathrm{H}, \mathrm{OCH}_{2} \mathrm{CH}_{3}\right)$. HRMS (M-t-Bu) ${ }^{+}$; Calcd for $\mathrm{C}_{10} \mathrm{H}_{18} \mathrm{NO}_{4}: 216.1236$; Found: 216.12381.

1-(tert-Butoxycarbonyl)-3-butylaziridine-2-carbonylaminoacetophenone (9). Ethyl 3butylaziridine-2-carboxylate 4 was converted into the corresponding $N$-Boc-aziridinecarboxylic acid in the same manner as described for 5 in $71 \%$ yield. A stirred, cooled $\left(0^{\circ} \mathrm{C}\right)$ mixture of 1 -(tert-butoxycarbonyl)-3butylaziridinecarboxylic acid $(0.60 \mathrm{~g}, 2.5 \mathrm{mmol})$ and iso-butyl chloroformate $(0.34 \mathrm{~mL}, 2.8 \mathrm{mmol})$ in dry THF (25 mL) was treated with triethylamine $(1.0 \mathrm{~mL}, 7.5 \mathrm{mmol})$ and stirred at the same temperature and after $1 \mathrm{~h} \alpha$ amino-acetophenone hydrogen chloride $(0.47 \mathrm{~g}, 2.8 \mathrm{mmol})$ was added. The reaction mixture was set aside at 0 ${ }^{\circ} \mathrm{C}$ for $1 \mathrm{~h}$, and then allowed to attain room temperature overnight. The reaction mixture was treated with saturated aqueous $\mathrm{NH}_{4} \mathrm{Cl}$ solution $(10 \mathrm{~mL})$, water, and extracted with EtOAc $(3 \times 40 \mathrm{~mL})$. The combined organic extracts were washed with a $10 \%$ aqueous citric acid solution, saturated aqueous $\mathrm{NaHCO}_{3}$ solution, brine, dried $\left(\mathrm{MgSO}_{4}\right)$, filtered and concentrated in vacuo. Column chromatography (silica, hexane-EtOAc (3:1)) of the residue gave $9(0.55 \mathrm{~g}, 61 \%)$ : mp. $137-138{ }^{\circ} \mathrm{C} ; \mathrm{IR}\left(\mathrm{CCl}_{4}\right) 3400,2960,1720,1675,1480,1160 \mathrm{~cm}^{-1} ;{ }^{1} \mathrm{H} \mathrm{NMR}$ $\left(\mathrm{CDCl}_{3}\right) \delta 0.92\left(\mathrm{~m}, 3 \mathrm{H}, \mathrm{CH}_{3}\left(\mathrm{CH}_{2}\right)_{3}\right), 1.2-1.8\left(\mathrm{~m}, 15 \mathrm{H}, \mathrm{CH}_{3}\left(\mathrm{CH}_{2}\right)_{3}, \mathrm{t}-\mathrm{Bu}\right), 2.58-2.76(\mathrm{~m}, 1 \mathrm{H}, \mathrm{Bu}-\mathrm{CH}), 2.86$ 
(d, J = 2.8 Hz, 1H, NCHCO), $4.76\left(\mathrm{~d}, \mathrm{~J}=4.5 \mathrm{~Hz}, 2 \mathrm{H}, \mathrm{NHCH}_{2} \mathrm{CO}\right.$ ), 7.17 (br t, $\left.1 \mathrm{H}, \mathrm{NH}\right), 7.45-7.67$ (m, 3H, 3, 4, 5-Ph), 7.88-8.05 (m, 2H, 2, 6-Ph). Anal. Calcd. for $\mathrm{C}_{20} \mathrm{H}_{28} \mathrm{~N}_{2} \mathrm{O}_{4}: \mathrm{C}, 66.64 ; \mathrm{H}, 7.83 ; \mathrm{N}, 7.77$; Found: $\mathrm{C}$, $66.63 ; \mathrm{H}, 7.65 ; \mathrm{N}, 7.77$.

(6-Butyl-2,4-dioxo-1,3-diaza-bicyclo[3.1.0]hex-3-yl)acetophenone (13). A stirred solution of $N$-Boc-aziridinecarboxamide $9(0.30 \mathrm{~g}, 0.84 \mathrm{mmol})$ in dry THF $(25 \mathrm{~mL})$ was treated with a catalytic amount of potasium tert-butoxide and then set aside at room temperature for $6 \mathrm{~h}$, and then treated with a dilute citric acid solution in water, and extracted with EtOAc $(3 \times 40 \mathrm{~mL})$. The combined organic extracts were washed with saturated aqueous $\mathrm{NaHCO}_{3}$ solution, brine, dried $\left(\mathrm{MgSO}_{4}\right)$, filtered and concentrated in vacuo. Column chromatography of the residue (silica gel, hexane-EtOAc (3:1)) gave bicyclic aziridine $13(0.24 \mathrm{~g}, 99 \%)$ as an oil; IR $\left(\mathrm{CCl}_{4}\right) 2920,1790,1725,1700,1410,1220 \mathrm{~cm}^{-1} ;{ }^{1} \mathrm{H}$ NMR $\left(\mathrm{CDCl}_{3}\right) \delta 0.92\left(\mathrm{br} \mathrm{t}, 3 \mathrm{H}, \mathrm{CH}_{3}\left(\mathrm{CH}_{2}\right)_{3}\right), 1.1-1.9$ (m, 6H, $\left.\mathrm{CH}_{3}\left(\mathrm{CH}_{2}\right)_{3}\right), 3.08-3.30(\mathrm{~m}, 2 \mathrm{H}, \mathrm{CHNCH}), 4.80\left(\mathrm{~s}, 2 \mathrm{H}, \mathrm{NCH}_{2} \mathrm{Ph}\right), 7.32-7.80$ (m, 3H, 3, 4, 5-Ph), 7.80-8.02 (m, 2H, 2, 6-Ph). HRMS (M)+; Calcd. for $\mathrm{C}_{16} \mathrm{H}_{18} \mathrm{~N}_{2} \mathrm{O}_{3}$ : 286.1317; Found: 286.13169.

1-(tert-Butoxycarbonyl)-3-hexylaziridine-2-carbonylaminoacetic acid methyl ester (10). A stirred, cooled $\left(0^{\circ} \mathrm{C}\right)$ mixture of trans- 1-(tert-Butoxycarbonyl)-3-hexylaziridine-2-carboxylic acid and iso-butyl chloroformate $(0.35 \mathrm{~mL}, 2.9 \mathrm{mmol})$ in dry THF $(26 \mathrm{~mL})$ was treated with triethylamine $(1.1 \mathrm{~mL}, 7.8 \mathrm{mmol})$ and after $1 \mathrm{~h}$ with glycine methyl ester hydrogen chloride $(0.36 \mathrm{~g}, 2.9 \mathrm{mmol})$. The mixture was then stirred a further $1 \mathrm{~h}$ and then at room temperature overnight. The resulting mixture was treated with saturated aqueous $\mathrm{NH}_{4} \mathrm{Cl}$ solution $(10 \mathrm{~mL})$, extracted with EtOAc and the combined organic extracts were washed with dilute aqueous citric acid, saturated aqueous $\mathrm{NaHCO}_{3}$, brine, dried $\left(\mathrm{MgSO}_{4}\right)$, filtered, and concentrated in vacuo. Column chromatography of the residue (silica gel, hexane-EtOAc (2:1)) gave 10 as white crystals $(0.63 \mathrm{~g}, 71 \%)$ : $\mathrm{mp} .92$ $-93{ }^{\circ} \mathrm{C}$; IR $\left(\mathrm{CCl}_{4}\right) 3420,2930,1750,1725,1690,1368,1160 \mathrm{~cm}^{-1} ;{ }^{1} \mathrm{H} \mathrm{NMR}\left(\mathrm{CDCl}_{3}\right) \delta 0.88(\mathrm{brt}, 3 \mathrm{H}$, $\left.\mathrm{CH}_{3}\left(\mathrm{CH}_{2}\right)_{5}\right), 1.20-1.60\left(\mathrm{~m}, 19 \mathrm{H}, \mathrm{CH}_{3}\left(\mathrm{CH}_{2}\right)_{5}, \mathrm{t}-\mathrm{Bu}\right), 2.54-2.72(\mathrm{~m}, 1 \mathrm{H}, \mathrm{Hex}-\mathrm{CH}), 2.81(\mathrm{~d}, \mathrm{~J}=2.9 \mathrm{~Hz}, 1 \mathrm{H}$, $\mathrm{NCHCO}), 3.76\left(\mathrm{~s}, 3 \mathrm{H}, \mathrm{OCH}_{3}\right), 4.02+4.05\left(2 \mathrm{xd}, \mathrm{J}=5.4 \mathrm{~Hz}, 2 \mathrm{H}, \mathrm{NHCH}_{2} \mathrm{CO}\right), 6.6(\mathrm{br} \mathrm{t}, 1 \mathrm{H}, \mathrm{NH})$. Anal. Calcd. for $\mathrm{C}_{17} \mathrm{H}_{30} \mathrm{~N}_{2} \mathrm{O}_{5}$ : C, 59.63; H, 8.83; N, 8.18; Found: $\mathrm{C}, 59.74 ; \mathrm{H}, 8.95 ; \mathrm{N}, 8.08$.

(6-Hexyl-2,4-dioxo-1,3-diaza-bicyclo[3.1.0]hex-3-yl)acetic acid methyl ester (14). A stirred solution of $N$-Boc-aziridinecarboxamide $10(0.61 \mathrm{~g}, 1.8 \mathrm{mmol})$ in $\mathrm{MeOH}(52 \mathrm{~mL})$ was treated with a few drops of $\mathrm{NaOMe}(1.3 \mathrm{M}$ in $\mathrm{MeOH})$, stirred at room temperature overnight and then concentrated in vacuo. Column chromatography of the residue (silica, hexane-EtOAc (3:1)) gave $14(0.25 \mathrm{~g}, 52 \%)$ and unreacted 10 $(0.18 \mathrm{~g}, 30 \%)$ : oil; IR $\left(\mathrm{CCl}_{4}\right) 2930,1795,1760,1735,1415,1230 \mathrm{~cm}^{-1} ;{ }^{1} \mathrm{H}$ NMR $\left(\mathrm{CDCl}_{3}\right) \delta 0.89$ (br t, 3H, $\left.\mathrm{CH}_{3}\left(\mathrm{CH}_{2}\right)_{5}\right), 1.2-1.8\left(\mathrm{~m}, 10 \mathrm{H}, \mathrm{CH}_{3}\left(\mathrm{CH}_{2}\right)_{5}\right), 2.8-3.0(\mathrm{~m}, 1 \mathrm{H}, \mathrm{Hex}-\mathrm{CH}), 3.20(\mathrm{~d}, \mathrm{~J}=3.1 \mathrm{~Hz}, 1 \mathrm{H}, \mathrm{NCHCO})$, $3.76\left(\mathrm{~s}, 3 \mathrm{H}, \mathrm{OCH}_{3}\right), 4.15\left(\mathrm{~s}, 2 \mathrm{H}, \mathrm{NCH}_{2}\right)$. HRMS $(\mathrm{M}+1)^{+}$; Calcd. for $\mathrm{C}_{13} \mathrm{H}_{21} \mathrm{~N}_{2} \mathrm{O}_{4}$ : 269.1501; Found: 269.14999 .

trans- $N$-Benzyl-1-(tert-butoxycarbonyl)-3-hexylaziridine-2-carboxamide (11). Using the same procedure as for 9 , trans- $N$-Boc-3-hexylaziridine-2-carboxylic acid $(0.47 \mathrm{~g}, 1.7 \mathrm{mmol})$ was converted into 11 using iso-butyl chloroformate $(0.26 \mathrm{~mL}, 2.0 \mathrm{mmol})$ as a condensing agent $(0.61 \mathrm{~g}, 99 \%$ yield $)$ : oil; IR $\left(\mathrm{CCl}_{4}\right)$ $3400,2920,1725,1685,1550,1250 \mathrm{~cm}^{-1} ;{ }^{1} \mathrm{H}$ NMR $\left(\mathrm{CDCl}_{3}\right) \delta$ 0.78-1.0 (br t, $\left.3 \mathrm{H}, \mathrm{CH}_{3}\left(\mathrm{CH}_{2}\right)_{5}\right), 1.18-1.75$ (m, 19H, $\left.\mathrm{CH}_{3}\left(\mathrm{CH}_{2}\right)_{5}, \mathrm{t}-\mathrm{Bu}\right), 2.48-2.69(\mathrm{~m}, 1 \mathrm{H}, \mathrm{Hex}-\mathrm{CH}), 2.82(\mathrm{~d}, \mathrm{~J}=3.0 \mathrm{~Hz}, 1 \mathrm{H}, \mathrm{NCHCO}), 4.42+4.44$ $\left(2 \mathrm{xd}, \mathrm{J}=5.9 \mathrm{~Hz}, 2 \mathrm{H}, \mathrm{NHCH}_{2}\right), 6.48(\mathrm{br} \mathrm{t}, 1 \mathrm{H}, \mathrm{NH}), 7.16-7.50(\mathrm{~m}, 5 \mathrm{H}, \mathrm{Ph})$. HRMS $(\mathrm{M}+1)^{+}$; Calcd. for $\mathrm{C}_{21} \mathrm{H}_{37} \mathrm{~N}_{2} \mathrm{O}_{3}: 361.2491$; Found: 361.24992 .

(6-Hexyl-2,4-dioxo-1,3-diaza-bicyclo[3.1.0]hex-3-yl)methylbenzene (15). A stirred, cooled $\left(0^{\circ} \mathrm{C}\right)$ solution of $N$-Boc-aziridinecarboxamide $11(0.35 \mathrm{~g}, 0.97 \mathrm{mmol})$ in dry THF $(10 \mathrm{~mL})$ was treated with a 
catalytic amount of potassium tert-butoxide, stirred at $0^{\circ} \mathrm{C}$ for $2.5 \mathrm{~h}$, and then at room temperature overnight. The reaction mixture was treated with a $10 \%$ aqueous solution of citric acid $(10 \mathrm{~mL})$, extracted with EtOAc $(3 \times 25$ $\mathrm{mL}$ ), and the combined organic extracts washed with $10 \%$ aqueous citric acid, aqueous saturated $\mathrm{NaHCO}_{3}$ and brine, dried $\left(\mathrm{MgSO}_{4}\right)$, filtered and concentrated in vacuo. Column chromatography of the residue (silica, hexaneEtOAc (7:1)) gave 15 as a white solid $(0.18 \mathrm{~g}, 65 \%)$, which was, then recrystallized from pet.-ether (60-80): $\mathrm{mp}$. $55-56{ }^{\circ} \mathrm{C}$; IR $\left(\mathrm{CCl}_{4}\right) 2920,1785,1720,1390,1340 \mathrm{~cm}^{-1} ;{ }^{1} \mathrm{H}$ NMR $\left(\mathrm{CDCl}_{3}\right) \delta 0.72-1.08(\mathrm{br} \mathrm{t}, 3 \mathrm{H}$, $\left.\mathrm{CH}_{3}\left(\mathrm{CH}_{2}\right)_{5}\right), 1.08-1.80\left(\mathrm{~m}, 10 \mathrm{H}, \mathrm{CH}_{3}\left(\mathrm{CH}_{2}\right)_{5}\right), 2.36-2.60(\mathrm{~m}, 1 \mathrm{H}, \mathrm{Hex}-\mathrm{CH}), 3.10(\mathrm{~d}, \mathrm{~J}=3.0 \mathrm{~Hz}, 1 \mathrm{H}$, $\mathrm{NCHCO}$ ), 4.52 (s, $2 \mathrm{H}, \mathrm{NCH}_{2} \mathrm{Ph}$ ), 7.20-7.60 (m, $5 \mathrm{H}, \mathrm{Ph}$ ). Anal. Calcd. for $\mathrm{C}_{17} \mathrm{H}_{22} \mathrm{~N}_{2} \mathrm{O}_{2}: \mathrm{C}, 71.30 ; \mathrm{H}, 7.74$; N, 9.78; Found: C, 71.42; H, 7.56; N, 9.61.

Methyl trans-1-(tert-butoxycarbonyl)-3-benzylaziridine-2-carboxylate 4 Oil; IR $\left(\mathrm{CCl}_{4}\right) 2590$, $1750,1730,1370,1315,1155 \mathrm{~cm}^{-1} ;{ }^{1} \mathrm{H}$ NMR $\left(\mathrm{CDCl}_{3}\right) \delta 1.43(\mathrm{~s}, 9 \mathrm{H}, \mathrm{t}-\mathrm{Bu}), 2.69-3.15(\mathrm{~m}, 4 \mathrm{H}, \mathrm{CHNCH}$, $\mathrm{CH}_{2} \mathrm{Ph}$ ), 3.73 (s, 3H, $\mathrm{OCH}_{3}$ ), 7.20-7.35 (m, 5H, Ph). HRMS (M)+; Calcd. for $\mathrm{C}_{15} \mathrm{H}_{21} \mathrm{NO}_{4}$ : 291.1470; Found: 291.14695 .

trans-1-(tert-Butoxycarbonyl)-3-benzylaziridine-2-carbonylaminoacetophenone (12). Using a similar procedure as described for $9, \mathrm{~N}$-Boc-aziridinecarboxamide 12 was prepared from $\mathrm{N}$-Boc aziridine methyl ester $(0.68 \mathrm{~g}, 2.3 \mathrm{mmol})$ from the corresponding carboxylic acid $(0.57 \mathrm{~g}, 89 \%$ yield $)$ using isobutyl chloroformate as the condensing agent in $61 \%$ total yield: $\mathrm{mp} .135 .5-136.5{ }^{\circ} \mathrm{C}$ : $\mathrm{IR}\left(\mathrm{CCl}_{4}\right) 3310,1700$, $1650,1565,1320 \mathrm{~cm}^{-1} ;{ }^{1} \mathrm{H} \mathrm{NMR}\left(\mathrm{CDCl}_{3}\right) \delta 1.0-1.8(\mathrm{~s}, 9 \mathrm{H}, \mathrm{t}-\mathrm{Bu}), 2.7-3.2\left(\mathrm{~m}, 4 \mathrm{H}, \mathrm{CHNCH}, \mathrm{CH}_{2} \mathrm{Ph}\right), 4.76$ (d, J = 4.5 Hz, 2H, $\mathrm{CH}_{2} \mathrm{COPh}$ ), 7.0-7.7 (m, 9H, NH, aromatic-H), 7.8-8.1 (m, 2H, Ph). Anal. Calcd. for $\mathrm{C}_{23} \mathrm{H}_{26} \mathrm{~N}_{2} \mathrm{O}_{4}$ : C, 70.03; H, 6.64; N, 7.10; Found: C, 69.73; H, 6.62; N, 7.22.

[6-(Benzyl)-2,4-dioxo-1,3-diaza-bicyclo[3.1.0]hex-3-yl]acetophenone (16). To a stirred cooled $\left(0^{\circ} \mathrm{C}\right)$ solution of $N$-Boc-aziridinecarboxamide $12(0.34 \mathrm{~g}, 0.86 \mathrm{mmol})$ in dry THF ( $\left.25 \mathrm{~mL}\right)$ was added a catalytic amount of potassium tert-butoxide. The mixture was stirred at $0^{\circ} \mathrm{C}$ for $3.5 \mathrm{~h}$, then at room temperature for $2.5 \mathrm{~h}$. The reaction mixture was treated with a $10 \%$ aqueous citric acid solution, extracted with EtOAc $(3 \times 40$ $\mathrm{mL}$ ) and the combined organic extracts washed with saturated aqueous $\mathrm{NaHCO}_{3}$ and brine, dried $\left(\mathrm{MgSO}_{4}\right)$, filtered, and concentrated in vacuo. Column chromatography of the residue (silica, hexane-EtOAc (3:1)) gave 16 $(0.24 \mathrm{~g}, 87 \%)$ : oil; IR $\left(\mathrm{CCl}_{4}\right) 3015,2930,1790,1725,1700,1410,1220 \mathrm{~cm}^{-1} ;{ }^{1} \mathrm{H} \mathrm{NMR}\left(\mathrm{CDCl}_{3}\right) \delta 3.03$ (d, J $\left.=5.4 \mathrm{~Hz}, 2 \mathrm{H}, \mathrm{CHCH}_{2} \mathrm{Ph}\right), 3.24(\mathrm{~d}, \mathrm{~J}=2.6 \mathrm{~Hz}, 1 \mathrm{H}, \mathrm{NCHCO}), 3.32-3.57\left(\mathrm{~m}, 1 \mathrm{H}, \mathrm{CHCH}_{2} \mathrm{Ph}\right), 4.78(\mathrm{~s}, 2 \mathrm{H}$, COC $\left.H_{2} \mathrm{Ph}\right), 7.20-7.76(\mathrm{~m}, 8 \mathrm{H}, 2 \mathrm{xPh}), 7.76-8.00(\mathrm{~m}, 2 \mathrm{H}, \mathrm{Ph})$. HRMS (M)+; Calcd. for $\mathrm{C}_{19} \mathrm{H}_{16} \mathrm{~N}_{2} \mathrm{O}_{3}$ : 320.1161 ; Found: 320.11574 .

Methyl cis-3-hexylaziridine-2-carboxylate. Methyl cis-3-hexyloxiranecarboxylate was prepared according to a literature method via hydrogenation using the Lindlar catalyst: 4,21 oil; $I R\left(\mathrm{CCl}_{4}\right) 2920,1760$, $1735,1440,1200 \mathrm{~cm}^{-1}$; ${ }^{1} \mathrm{H}$ NMR $\left(\mathrm{CDCl}_{3}\right) \delta 0.88\left(\mathrm{~m}, 3 \mathrm{H}, \mathrm{CH}_{3}\left(\mathrm{CH}_{2}\right)_{5}\right), 1.1-1.8\left(\mathrm{~m}, 10 \mathrm{H}, \mathrm{CH}_{3}\left(\mathrm{CH}_{2}\right)_{5}\right), 3.1-$ $3.3(\mathrm{~m}, 1 \mathrm{H}, \mathrm{Hex}-\mathrm{CHO}), 3.54(\mathrm{~d}, \mathrm{~J}=4.5 \mathrm{~Hz}, 1 \mathrm{H}, \mathrm{OCHCO}), 3.80\left(\mathrm{~s}, 3 \mathrm{H}, \mathrm{OCH}_{3}\right)$. A solution of methyl cis-3hexyloxiranecarboxylate $(1.1 \mathrm{~g}, 5.9 \mathrm{mmol}), \mathrm{NaN}_{3}(1.2 \mathrm{~g}, 18 \mathrm{mmol})$, and $\mathrm{NH}_{4} \mathrm{Cl}(0.94 \mathrm{~g}, 18 \mathrm{mmol})$ in $\mathrm{MeOH}$ $(28 \mathrm{~mL}$ ) was heated under reflux for $24 \mathrm{~h}$, and then concentrated in vacuo. The residue was triturated with ether and brine. The organic layer was washed with brine, dried $\left(\mathrm{MgSO}_{4}\right)$, filtered and concentrated in vacuo. Column chromatography of the residue (silica, hexane-EtOAc (4:1)) gave an inseparable mixture of two isomers $(1.2 \mathrm{~g}$, $89 \%$, ratio $3.6: 1$ by GC). The mixture of the isomers $(0.40 \mathrm{~g}, 1.7 \mathrm{mmol})$ in $N, N$-dimethylformamide $(40 \mathrm{~mL})$ was treated with $\mathrm{PPh}_{3}(0.50 \mathrm{~g}, 1.9 \mathrm{mmol})$ at room temperature with stirring. The mixture was stirred for 3 days at room temperature and then heated under reflux for $4 \mathrm{~h}$. The cooled reaction mixture was diluted with ether ( 150 $\mathrm{mL}$ ), and the organic solution washed with brine, dried $\left(\mathrm{MgSO}_{4}\right)$, filtered and concentrated in vacuo. Column 
chromatography of the residue (silica, hexane-EtOAc (3:1)) gave methyl cis-3-hexylaziridine-2-carboxylate (0.08 $\mathrm{g}, 25 \%)$ : oil; IR $\left(\mathrm{CCl}_{4}\right) 3260,2920,1725,1440,1380,1200 \mathrm{~cm}^{-1} ;{ }^{1} \mathrm{H} \mathrm{NMR}\left(\mathrm{CDCl}_{3}\right) \delta 0.88$ (br t, 3H, $\left.\mathrm{CH}_{3}\left(\mathrm{CH}_{2}\right)_{5}\right), 1.1-1.7\left(\mathrm{~m}, 11 \mathrm{H}, \mathrm{NH}, \mathrm{CH}_{3}\left(\mathrm{CH}_{2}\right)_{5}\right), 2.1-2.3(\mathrm{~m}, 1 \mathrm{H}, \mathrm{Hex}-\mathrm{CH}), 2.66(\mathrm{~d}, \mathrm{~J}=6 \mathrm{~Hz}, 1 \mathrm{H}, \mathrm{NCH})$, 3.77 (s, $3 \mathrm{H}, \mathrm{OCH}_{3}$ ). HRMS (M+1)+; Calcd. for $\mathrm{C}_{15} \mathrm{H}_{28} \mathrm{NO}_{4}$ : 286.2018; Found: 286.20194 .

Methyl cis-1-(tert-butoxycarbonyl)-3-hexylaziridine-2-carboxylate. Using the standard procedure, $c i s-N$-Boc-aziridinecarboxylic acid methyl ester was obtained in $65 \%$ yield: oil; IR $\left(\mathrm{CCl}_{4}\right) 2920$, $1755,1720,1545,1250,1000 \mathrm{~cm}^{-1}$; ${ }^{1} \mathrm{H}$ NMR $\left(\mathrm{CDCl}_{3}\right) \delta 0.88$ (br t, $\left.3 \mathrm{H}, \mathrm{CH}_{3}\left(\mathrm{CH}_{2}\right)_{5}\right), 1.1-1.7(\mathrm{~m}, 19 \mathrm{H}$, $\left.\mathrm{CH}_{3}\left(\mathrm{CH}_{2}\right)_{5}, \mathrm{t}-\mathrm{Bu}\right), 2.5-2.7(\mathrm{~m}, 1 \mathrm{H}, \mathrm{Hex}-\mathrm{CH}), 3.12(\mathrm{~d}, \mathrm{~J}=6.7 \mathrm{~Hz}, 1 \mathrm{H}, \mathrm{NCH}), 3.77\left(\mathrm{~s}, 3 \mathrm{H}, \mathrm{OCH}_{3}\right)$. HRMS $(\mathrm{M}+1)^{+}$; Calcd. for $\mathrm{C}_{21} \mathrm{H}_{37} \mathrm{~N}_{2} \mathrm{O}_{7}: 429.2601$; Found: 429.25973 .

cis-1-(tert-Butoxycarbonyl)-3-hexylaziridine-2-carbonylamino-malonic acid diethyl ester (17). $\mathrm{Cis}$ - $\mathrm{N}$-Boc-aziridinecarboxylic acid methyl ester was converted into the cis-aziridinecarboxamide 17 in $45 \%$ yield by a method similar to that used for trans- $\mathrm{N}$-Boc-aziridinecarboxamide 5: oil; IR $\left(\mathrm{CCl}_{4}\right) 3420,2930$, $1760,1725,1500,1160 \mathrm{~cm}^{-1} ;{ }^{1} \mathrm{H}$ NMR $\left(\mathrm{CDCl}_{3}\right) \delta 0.88\left(\mathrm{~m}, 3 \mathrm{H}, \mathrm{CH}_{3}\left(\mathrm{CH}_{2}\right)_{5}\right) 1.1-1.8\left(\mathrm{~m}, 25 \mathrm{H}, \mathrm{CH}_{3}\left(\mathrm{CH}_{2}\right)_{5}\right.$, t$\left.\mathrm{Bu}, 2 \mathrm{xOCH}_{2} \mathrm{CH}_{3}\right), 2.46-2.72(\mathrm{~m}, 1 \mathrm{H}, \mathrm{Hex}-\mathrm{CH}), 3.04-3.25(\mathrm{~m}, 1 \mathrm{H}, \mathrm{NCH}), 4.27(\mathrm{q}, \mathrm{J}=7.1 \mathrm{~Hz}, 4 \mathrm{H}$, $2 \times \mathrm{OCH}_{2} \mathrm{CH}_{3}$ ), 5.01-5.19 (br d, $\left.1 \mathrm{H}, \mathrm{CH}\left(\mathrm{CO}_{2} \mathrm{Et}\right)_{2}\right), 7.4$ (br d, 1H, NH). HRMS (M+1)+; Calcd. for $\mathrm{C}_{21} \mathrm{H}_{37} \mathrm{~N}_{2} \mathrm{O}_{7}: 429.2601$; Found: 429.25973.

\section{3,4-cis-3-(tert-Butoxycarbonyl)amino-4-hexyl-5,5-di(ethoxycarbonyl)pyrrolidin-2-one}

(18). A stirred cooled $\left(0^{\circ} \mathrm{C}\right)$ solution of aziridine $5(0.64 \mathrm{~g}, 1.5 \mathrm{mmol})$ in acetone $(15 \mathrm{~mL})$ was treated with hydriodic acid $(1.5 \mathrm{mmol})$. The mixture was stirred for $3 \mathrm{~h}$ at this temperature, then treated with water. The solid produced was filtered and the filtrate cake washed with water. The solid was dried in vacuo to give the crude product $(0.49 \mathrm{~g}, 59 \%)$. The crude product without further purification was dissolved in absolute ethanol $(15 \mathrm{~mL})$ and treated with sodium ethoxide/ethanol $(1.3 \mathrm{mmol})$ and stirred overnight at room temperature. The resultant mixture was treated with saturated aqueous $\mathrm{NH}_{4} \mathrm{Cl}$ solution $(30 \mathrm{~mL})$, concentrated in vacuo and the residue extracted with EtOAc $(3 x 40 \mathrm{~mL})$. The combined organic extracts were washed with brine, dried $\left(\mathrm{MgSO}_{4}\right)$, filtered and concentrated in vacuo. Column chromatography of the residue (silica, hexane-EtOAc (2:1) to (1:1)) gave cis-pyrrolidinone $18(0.10 \mathrm{~g}, 16 \%)$ and trans-pyrrolidinone $7(0.09 \mathrm{~g}, 15 \%)$ : mp. 109-1 $10^{\circ} \mathrm{C}$; $\mathrm{IR}\left(\mathrm{CCl}_{4}\right)$ $3420,2920,1705,1485,1365 \mathrm{~cm}^{-1} ;{ }^{1} \mathrm{H} \mathrm{NMR}\left(\mathrm{CDCl}_{3}\right) \delta 0.87\left(\mathrm{t}, \mathrm{J}=6.4 \mathrm{~Hz}, 3 \mathrm{H}, \mathrm{CH}_{3}\left(\mathrm{CH}_{2}\right)_{5}\right), 1.2-1.6(\mathrm{~m}$, $\left.25 \mathrm{H}, \mathrm{CH}_{3}\left(\mathrm{CH}_{2}\right)_{5}, \mathrm{t}-\mathrm{Bu}, 2 \mathrm{OCCH}_{2} \mathrm{CH}_{3}\right), 2.9-3.0(\mathrm{~m}, 1 \mathrm{H}, \mathrm{Hex}-\mathrm{CH}), 4.2-4.3\left(\mathrm{~m}, 4 \mathrm{H}, 2 \mathrm{XOCH}_{2} \mathrm{CH}_{3}\right), 4.58$ (dd, J $=8.4,9.2 \mathrm{~Hz}, 1 \mathrm{H}, \mathrm{CHNH}), 5.23(\mathrm{~d}, \mathrm{~J}=9.2 \mathrm{~Hz}, 1 \mathrm{H}, \mathrm{CHNH}), 7.13(\mathrm{br} \mathrm{s}, 1 \mathrm{H}, \mathrm{NHCO})$; Anal. Calcd. for $\mathrm{C}_{21} \mathrm{H}_{36} \mathrm{~N}_{2} \mathrm{O}_{7}: \mathrm{C}, 58.85 ; \mathrm{H}, 8.48 ; \mathrm{N}, 6.54$. Found: $\mathrm{C}, 58.41 ; \mathrm{H}, 8.64 ; \mathrm{N}, 6.43$.

trans-3-Hexyloxirane-2-carbonylaminomalonic acid diethyl ester (19). Ethyl trans-3hexyloxirane-2-carboxylate $5(2.0 \mathrm{~g}, 10 \mathrm{mmol})$ in $\mathrm{EtOH}(100 \mathrm{~mL})$ and $\mathrm{H}_{2} \mathrm{O}(90 \mathrm{~mL})$ was heated under reflux for $5 \mathrm{~h}$ in the presence of $\mathrm{NaOH}(0.80 \mathrm{~g}, 20 \mathrm{mmol})$. The reaction mixture was cooled to room temperature, concentrated in vacuo, then acidified to $\mathrm{pH} 3$ with dilute $\mathrm{H}_{2} \mathrm{SO}_{4}$ and extracted with ether $(3 \times 50 \mathrm{~mL})$. The organic layer was extracted with $15 \% \mathrm{NaOH}$ solution and the aqueous alkaline solution acidified to $\mathrm{pH} 3$ with dilute $\mathrm{H}_{2} \mathrm{SO}_{4}$, and the mixture was then extracted with ether $(3 \mathrm{x} 50 \mathrm{~mL})$. The combined organic extracts were dried $\left(\mathrm{MgSO}_{4}\right)$, filtered and concentrated in vacuo to give trans-3-hexyloxirane-2-carboxylic acid $(1.7 \mathrm{~g}, 99 \%)$. A solution of the crude product $(1.7 \mathrm{~g}, 9.9 \mathrm{mmol})$ and diethyl aminomalonate $(2.3 \mathrm{~g}, 11 \mathrm{mmol})$ in EtOAc $(80 \mathrm{~mL})$ was treated with dicyclohexylcarbodiimide (DCC) $(2.2 \mathrm{~g}, 11 \mathrm{mmol})$ at $0{ }^{\circ} \mathrm{C}$ for $1 \mathrm{~h}$ and overnight at room temperature. Then the white precipitate was filtered and washed with EtOAc $(5 \times 20 \mathrm{~mL})$, and the filtrate and the washings were combined and washed with saturated aqueous $\mathrm{NaHCO}_{3}$ solution, a $10 \%$ aqueous citric acid solution, brine, dried $\left(\mathrm{MgSO}_{4}\right)$, filtered and concentrated in vacuo. Column chromatography (silica, hexane- 
EtOAc (5:1)) of the residue gave $19(2.9 \mathrm{~g}, 88 \%)$ : mp. $49-50^{\circ} \mathrm{C}$; IR $\left(\mathrm{CCl}_{4}\right) 3400,2930,1760,1690,1500$, $1370 \mathrm{~cm}^{-1}$; ${ }^{\mathrm{H}} \mathrm{NMR}\left(\mathrm{CDCl}_{3}\right) \delta 1.75-1.01\left(\mathrm{~m}, 3 \mathrm{H}, \mathrm{CH}_{3}\left(\mathrm{CH}_{2}\right) 5\right), 1.1-1.8\left(\mathrm{~m}, 16 \mathrm{H}, \mathrm{CH}_{3}\left(\mathrm{CH}_{2}\right)_{5}, 2 \mathrm{xOCH}_{2} \mathrm{CH}_{3}\right)$, 2.96-3.19 (m, 1H, Hex-CH), $3.27(\mathrm{~d}, \mathrm{~J}=2 \mathrm{~Hz}, 1 \mathrm{H}, \mathrm{OCHCO}), 4.27+4.29(2 \mathrm{xq}, \mathrm{J}=7.1 \mathrm{~Hz}, 4 \mathrm{H}$, $\left.2 \times \mathrm{OCH}_{2} \mathrm{CH}_{3}\right), 5.14\left(\mathrm{~d}, \mathrm{~J}=5.6 \mathrm{~Hz}, 1 \mathrm{H}, \mathrm{CH}\left(\mathrm{CO}_{2} \mathrm{Et}\right)_{2}\right), 7.04(\mathrm{~d}, \mathrm{~J}=7.6 \mathrm{~Hz}, 1 \mathrm{H}, \mathrm{NH})$. Anal. Calcd. for $\mathrm{C}_{16} \mathrm{H}_{27} \mathrm{NO}_{6}$ : C, 58.34; $\mathrm{H}, 8.26 ; \mathrm{N}, 4.25$; Found: $\mathrm{C}, 58.81 ; \mathrm{H}, 8.33 ; \mathrm{N}, 4.33$.

trans-5,5-Di(ethoxycarbonyl)-3-hydroxy-4-hexylpyrrolidin-2-one (20). A stirred solution of the oxiranecarboxamide $19(0.40 \mathrm{~g}, 1.2 \mathrm{mmol})$ in $\mathrm{EtOH}(36 \mathrm{~mL})$ under argon was treated with a catalytic amount of $\mathrm{NaOE} / \mathrm{EtOH}$, set aside overnight at room temperature, and then concentrated in vacuo. Column chromatography (silica, hexane-EtOAc (3:1)) of the residue gave $19(0.09 \mathrm{~g}, 22 \%)$ and $20(0.31 \mathrm{~g}, 78 \%)$ : bp. $227^{\circ} \mathrm{C} / 2.0 \mathrm{~mm} \mathrm{Hg}$; IR $\left(\mathrm{CCl}_{4}\right) 3540,3430,2930,1740,1250,1095 \mathrm{~cm}^{-1} ;{ }^{1} \mathrm{H}$ NMR $\left(\mathrm{CDCl}_{3}\right) \delta$ 0.75-1.08 (br t, $\left.3 \mathrm{H}, \mathrm{CH}_{3}\left(\mathrm{CH}_{2}\right)_{5}\right), 1.10-2.10\left(\mathrm{~m}, 16 \mathrm{H}, \mathrm{CH}_{3}\left(\mathrm{CH}_{2}\right)_{5}, 2 \mathrm{xOCH}_{2} \mathrm{CH}_{3}\right), 2.55-2.86$ (m, $1 \mathrm{H}, \mathrm{Hex}-\mathrm{CH}$ ), 3.86 (br s, $1 \mathrm{H}, \mathrm{OH}), 4.21(\mathrm{~d}, \mathrm{~J}=7 \mathrm{~Hz}, 1 \mathrm{H}, \mathrm{CHOH}), 4.27+4.31\left(2 \times \mathrm{xq}, \mathrm{J}=7.0 \mathrm{~Hz}, 4 \mathrm{H}, 2 \times \mathrm{OCH}_{2} \mathrm{CH}_{3}\right), 7.06(\mathrm{br} \mathrm{s}, 1 \mathrm{H}$, $\mathrm{NH}$ ). Anal. Calcd. for $\mathrm{C}_{16} \mathrm{H}_{27} \mathrm{NO}_{6} ; \mathrm{C}, 58.34 ; \mathrm{H}, 8.26 ; \mathrm{N}, 4.25$; Found: $\mathrm{C}, 58.80 ; \mathrm{H}, 8.18 ; \mathrm{N}, 4.67$. HRMS $(\mathrm{M}+1)^{+}$; Calcd. for $\mathrm{C}_{16} \mathrm{H}_{28} \mathrm{NO}_{6}$ : 330.1916 ; Found: 330.19166.

$\left.\mathrm{C}_{6} \mathrm{H}_{4} \mathrm{OCH}_{3}\right), 7.27\left(\mathrm{~d}, \mathrm{~J}=9.0 \mathrm{~Hz}, 2 \mathrm{H}, 2,6-\mathrm{C}_{6} \mathrm{H}_{4} \mathrm{OCH}_{3}\right), 7.39\left(\mathrm{~d}, \mathrm{~J}=9.0 \mathrm{~Hz}, 2 \mathrm{H}, 3,5-\mathrm{C}_{6} \mathrm{H}_{4} \mathrm{CH}_{3}\right), 7.75$ (d. J $=8.1 \mathrm{~Hz}, 2 \mathrm{H}, 2,6-\mathrm{C}_{6} \mathrm{H}_{4} \mathrm{CH}_{3}$ ). HRMS (M)+; Calcd. for $\mathrm{C}_{30} \mathrm{H}_{40} \mathrm{O}_{8} \mathrm{~N}_{2} \mathrm{~S}: 588.2505$; Found: 588.2503 .

trans-3-Butyl-oxirane-2-carbonyl-( $N$-methyl)aminomalonic acid diethyl ester (21). Ethyl trans-3-butyloxirane-carboxylate $(0.85 \mathrm{~g}, 4.9 \mathrm{mmol})$ was hydrolysed with $\mathrm{LiOH} / \mathrm{H}_{2} \mathrm{O}(1.0 \mathrm{M})$ using the standard method as described for (5). A stirred solution of the oxiranecarboxylic acid $(0.60 \mathrm{~g}, 4.2 \mathrm{mmol}, 85 \%$ yield) thus obtained, diethyl (methylamino)malonate $(0.79 \mathrm{~g}, 4.2 \mathrm{mmol})$ and 1-hydroxybenzotriazole $(0.64 \mathrm{~g}, 4.2 \mathrm{mmol})$ in EtOAc $(60 \mathrm{~mL})$ was treated with dicyclohexylcarbodiimide $(0.87 \mathrm{~g}, 4.2 \mathrm{mmol})$ at $0^{\circ} \mathrm{C}$ for $1 \mathrm{~h}$ and at room temperature overnight. After standard workup and column chromatography (silica, Hexane-EtOAc (3:1)), 21 $(0.50 \mathrm{~g}, 38 \%)$ was obtained: oil; IR $\left(\mathrm{CCl}_{4}\right) 2960,1740,1665,1465,1300 \mathrm{~cm}^{-1} ;{ }^{1} \mathrm{H}$ NMR $\left(\mathrm{CDCl}_{3}\right) \delta$ 0.8-1.1 (m, 3H, $\left.\mathrm{CH}_{3}\left(\mathrm{CH}_{2}\right)_{3}\right), 1.2-1.9\left(\mathrm{~m}, 12 \mathrm{H}, \mathrm{CH}_{3}\left(\mathrm{CH}_{2}\right)_{3}, 2 \mathrm{XOCH}_{2} \mathrm{CH}_{3}\right), 3.1-3.3(\mathrm{~m}, 1 \mathrm{H}, \mathrm{Bu}-\mathrm{CH}), 3.25(\mathrm{~s}, 3 \mathrm{H}$, $\left.\mathrm{NCH}_{3}\right), 3.48(\mathrm{~d}, \mathrm{~J}=2.0 \mathrm{~Hz}, 1 \mathrm{H}, \mathrm{OCHCO}), 4.1-4.4\left(\mathrm{~m}, 4 \mathrm{H}, 2 \mathrm{xOCH}_{2} \mathrm{CH}_{3}\right), 5.91\left(\mathrm{~s}, 1 \mathrm{H}, \mathrm{CH}\left(\mathrm{CO}_{2} \mathrm{Et}\right)_{2}\right)$. HRMS (M) ${ }^{+}$Calcd for $\mathrm{C}_{15} \mathrm{H}_{25} \mathrm{O}_{6} \mathrm{~N}$ : 315.16819 ; Found: 315.16821 .

trans-4-Butyl-5,5-di(ethoxycarbonyl)-3-hydroxy-1-methylpyrrolidin-2-one (22). The $\gamma$ lactam cyclization reaction was carried out following the standard procedure for intramolecular ring opening of aziridinecarboxamides (method A) using a catalytic amount of $\mathrm{NaOEt} / \mathrm{EtOH}$. After purification by column chromatography (silica, Hexane-EtOAc (2:1)), 22 was obtained in 93\% yield as an oil; IR $\left(\mathrm{CCl}_{4}\right) 3400,2960$, $1740,1720,1240 \mathrm{~cm}^{-1} ;{ }_{1} \mathrm{H}$ NMR $\left(\mathrm{CDCl}_{3}\right) \delta 0.91\left(\mathrm{t}, \mathrm{J}=7.2 \mathrm{~Hz}, 3 \mathrm{H}, \mathrm{CH}_{3}\left(\mathrm{CH}_{2}\right)_{3}\right), 1.25-1.35(\mathrm{~m}, 12 \mathrm{H}$, $\left.\mathrm{CH}_{3}\left(\mathrm{CH}_{2}\right)_{3}, 2 \times \mathrm{OCH}_{2} \mathrm{CH}_{3}\right), 2.6-2.7(\mathrm{~m}, 1 \mathrm{H}, \mathrm{Bu}-\mathrm{CH}), 2.95\left(\mathrm{~s}, 3 \mathrm{H}, \mathrm{NCH}_{3}\right), 3.7$ (br s, $\left.1 \mathrm{H}, \mathrm{OH}\right), 4.08(\mathrm{~d}, \mathrm{~J}=$ $10 \mathrm{~Hz}, 1 \mathrm{H}, \mathrm{CHOH}), 4.27\left(\mathrm{q}, \mathrm{J}=7.2 \mathrm{~Hz}, 2 \mathrm{H}, \mathrm{OCH}_{2} \mathrm{CH}_{3}\right), 4.32\left(\mathrm{q}, \mathrm{J}=7.2 \mathrm{~Hz}, 2 \mathrm{H}, \mathrm{OCH}_{2} \mathrm{CH}_{3}\right) . \mathrm{HRMS}$ $(\mathrm{M}+\mathrm{H})^{+}$Calcd. for $\mathrm{C}_{15} \mathrm{H}_{26} \mathrm{O}_{6} \mathrm{~N}: 316.17601$ : Found; 316.17618 . Anal. Calcd.: $\mathrm{C}, 56.95 ; \mathrm{H}, 8.28 ; \mathrm{N}, 4.43$; Found: $\mathrm{C}, 57.29 ; \mathrm{H}, 8.28 ; \mathrm{N}, 4.43$.

[trans-3-Hexyl-1-(toluene-4-sulfonyl)aziridine-2-carbonyl(N-benzyl)amino]acetic acid ethyl ester (23). Using the standard method as described for (5), by DCC coupling in the presence of 1 hydroxybenzotriazole $(0.37 \mathrm{~g}, 2.4 \mathrm{mmol})$, trans-1-(toluene-4-sulfonyl)-3-hexylaziridine-2-carboxylic acid $(0.79$ $\mathrm{g}, 2.4 \mathrm{mmol})$ was condensed with $N$-benzylglycine ethyl ester $(0.52 \mathrm{~g}, 2.6 \mathrm{mmol})$ to give $23(1.1 \mathrm{~g}, 87 \%)$ : oil; IR $\left(\mathrm{CCl}_{4}\right) 2920,1745,1670,1450,1330,1160 \mathrm{~cm}^{-1} ;{ }^{1} \mathrm{H}$ NMR $\left(\mathrm{CDCl}_{3}\right) \delta 0.7-1.08\left(\mathrm{br} \mathrm{t}, 3 \mathrm{H}, \mathrm{CH}_{3}\left(\mathrm{CH}_{2}\right)_{5}\right)$, 1.08-2.1 (m, 13H, $\left.\mathrm{CH}_{3}\left(\mathrm{CH}_{2}\right)_{5}, \mathrm{OCH}_{2} \mathrm{CH}_{3}\right), 2.41\left(\mathrm{~s}, 3 \mathrm{H}, \mathrm{PhCH}_{3}\right), 3.05-3.40(\mathrm{~m}, 1 \mathrm{H}, \mathrm{Hex}-\mathrm{CH}), 3.51(\mathrm{~d}, \mathrm{~J}=$ $4.2 \mathrm{~Hz}, 1 \mathrm{H}, \mathrm{NCHCO}), 3.85-4.30\left(\mathrm{~m}, 4 \mathrm{H}, \mathrm{CH}_{2} \mathrm{CO}_{2} \mathrm{Et}, \mathrm{OCH}_{2} \mathrm{CH}_{3}\right), 4.75\left(\mathrm{~d}, \mathrm{~J}=3 \mathrm{~Hz}, 2 \mathrm{H}, \mathrm{CH}_{2} \mathrm{Ph}\right), 7.00-7.46$ 
(m, 7H, $\left.\mathrm{CH}_{2} P h, 3,5-\mathrm{C}_{6} \mathrm{H}_{4} \mathrm{CH}_{3}\right), 7.70-7.94\left(\mathrm{~m}, 2 \mathrm{H}, 2,6-\mathrm{C}_{6} \mathrm{H}_{4} \mathrm{CH}_{3}\right.$ ). HRMS (M)+; Calcd. for $\mathrm{C}_{27} \mathrm{H}_{36} \mathrm{~N}_{2} \mathrm{O}_{5} \mathrm{~S}$ : 500.2345; Found: 500.2344. (24).

cis-1-Benzyl-[1-(toluene-4-sulfonylamino)-hept-1-yl]-4-ethoxycarbonylazetidin-2-one

Method A (with LDA): Lithium diisopropylamide ( $0.99 \mathrm{mmol}, 0.20 \mathrm{M}$ in THF) was added to a stirred solution of aziridine $23(0.45 \mathrm{~g}, 0.90 \mathrm{mmol})$ in dry THF $(25 \mathrm{~mL})$ under argon cooled to $-78{ }^{\circ} \mathrm{C}$. The reaction mixture was allowed to warm to $0^{\circ} \mathrm{C}$ and then stirred at the same temperature overnight. The reaction mixture was treated with $10 \%$ aqueous citric acid, extracted with EtOAc $(3 \times 30 \mathrm{~mL})$, the combined organic extracts washed with aqueous saturated $\mathrm{NaHCO}_{3}$, brine, dried $\left(\mathrm{MgSO}_{4}\right)$, filtered and concentrated in vacuo. Column chromatography (silica, hexane-EtOAc (3:1)) of the residue gave $23(0.24 \mathrm{~g})$ and azetidinone $24(0.03 \mathrm{~g}, 7 \%)$.

Method B (with tert-BuOK): Aziridinecarboxamide $23(0.34 \mathrm{~g}, 0.68 \mathrm{mmol})$ in dry THF ( $5 \mathrm{~mL}$ ) was added to a stirred cooled $\left(0^{\circ} \mathrm{C}\right)$ solution of tert-BuOK $(0.084 \mathrm{~g}, 0.75 \mathrm{mmol})$ in THF $(20 \mathrm{~mL})$ under argon, and then stirred overnight at $0^{\circ} \mathrm{C}$. The same workup as described in method $\mathrm{A}$ was followed to give $23(0.13 \mathrm{~g}, 38 \%)$ and 24 (0 $07 \mathrm{~g}, 19 \%$ ): oil; IR $\left(\mathrm{CCl}_{4}\right) 3340,2920,1745,1560,1350 \mathrm{~cm}^{-1} ;{ }^{1} \mathrm{H}$ NMR $\left(\mathrm{CDCl}_{3}\right) \delta 0.80-0.86(\mathrm{~m}, 3 \mathrm{H}$, $\left.\mathrm{CH}_{3}\left(\mathrm{CH}_{2}\right)_{5}\right), 1.05-1.6\left(\mathrm{~m}, 13 \mathrm{H}, \mathrm{CH}_{3}\left(\mathrm{CH}_{2}\right)_{5}, \mathrm{OCH}_{2} \mathrm{CH}_{3}\right), 2.41$ (s, 3H, $\left.\mathrm{C}_{6} \mathrm{H}_{4} \mathrm{CH}_{3}\right), 3.61$ (dd, J = 4.7, $5.9 \mathrm{~Hz}$, $1 \mathrm{H}, \mathrm{CHCONBn}), 3.69-3.78(\mathrm{~m}, 1 \mathrm{H}, \mathrm{CHNH}), 3.95(\mathrm{~d}, \mathrm{~J}=5.9 \mathrm{~Hz}, 1 \mathrm{H}, \mathrm{CHCO}), 4.13+4.27(2 \mathrm{~s}, 1 \mathrm{H}$, $\mathrm{CH} H \mathrm{Ph}), 4.27+4.31\left(2 \mathrm{q}, \mathrm{J}=4.3 \mathrm{~Hz}, 2 \mathrm{H}, \mathrm{OCH}_{2} \mathrm{CH}_{3}\right), 4.77+4.92(2 \mathrm{~s}, 1 \mathrm{H}, \mathrm{CHHPh}), 5.13(\mathrm{~d}, \mathrm{~J}=9.4 \mathrm{~Hz}, 1 \mathrm{H}$, $\mathrm{NH})$, 7.18-7.21 (m, $\left.2 \mathrm{H}, \mathrm{CH}_{2} P h\right), 7.26-7.35\left(\mathrm{~m}, 5 \mathrm{H}, \mathrm{CH}_{2} \mathrm{Ph}, 3,5-\mathrm{C}_{6} \mathrm{H}_{4} \mathrm{CH}_{3}\right), 7.73-7.76(\mathrm{~m}, 2 \mathrm{H}, 2,6-$ $\mathrm{C}_{6} \mathrm{H}_{4} \mathrm{CH}_{3}$ ). HRMS (M)+; Calcd. for $\mathrm{C}_{27} \mathrm{H}_{36} \mathrm{~N}_{2} \mathrm{O}_{5} \mathrm{~S}: 500.2345$; Found: 500.2345 .

3-(tert-Butoxycarbonylamino)-4-hexyl-5-ethoxycarbonylpyrrolidin-2-one (26). A mixture of diester $7(0.30 \mathrm{~g}, 0.70 \mathrm{mmol})$, lithium bromide $(0.061 \mathrm{~g}, 0.70 \mathrm{mmol})$, water $(0.013 \mathrm{~g}, 0.70 \mathrm{mmol})$ in dimethyl-2-imidazolidinone (DMI) $(7 \mathrm{~mL})$ was heated at $155^{\circ} \mathrm{C}$ for $4 \mathrm{~h}$. The cooled mixture was poured into ice/water and extracted with EtOAc $(2 \times 20 \mathrm{~mL})$. The combined organic extracts were dried $\left(\mathrm{MgSO}_{4}\right)$, filtered and concentrated in vacuo. Column chromatography (silica, hexane-EtOAc (1:1)) of the residue gave an inseparable mixture (1:1 ratio) of monoester $26(0.16 \mathrm{~g}, 64 \%)$ as an oil: $\mathrm{IR}\left(\mathrm{CCl}_{4}\right) 3300,1720,1490,1365,1170 \mathrm{~cm}^{-1} ;{ }^{1} \mathrm{H}$ NMR $\left(\mathrm{CDCl}_{3}\right) \delta 0.88\left(\mathrm{t}, \mathrm{J}=6 \mathrm{~Hz}, 3 \mathrm{H}, \mathrm{CH}_{3}\left(\mathrm{CH}_{2}\right)_{5}\right), 1.2-1.5\left(\mathrm{~m}, 2 \mathrm{H}, \mathrm{t}-\mathrm{Bu}, \mathrm{OCH}_{2} \mathrm{CH}_{3}, \mathrm{CH}_{3}\left(\mathrm{CH}_{2}\right)_{4} \mathrm{CH}_{2}\right)$, 1.6-1.8 (m, $\left.2 \mathrm{H}, \mathrm{CH}_{3}\left(\mathrm{CH}_{2}\right)_{4} \mathrm{CH}_{2}\right), 2.2-2.3(\mathrm{~m}, 0.5 \mathrm{H}, \mathrm{Hex}-\mathrm{CH}), 2.4-2.6(\mathrm{~m}, 0.5 \mathrm{H}, \mathrm{Hex}-\mathrm{CH}), 3.83(\mathrm{~d}, \mathrm{~J}=7.6$ $\mathrm{Hz}, 0.5 \mathrm{H}, \mathrm{CHNHBOc}), 4.16-4.28\left(\mathrm{~m}, 3.5 \mathrm{H}, \mathrm{NHCHCO}_{2} \mathrm{Et}, \mathrm{CHCHNHBoc}, \mathrm{OCH}_{2} \mathrm{CH}_{3}\right), 5.06(\mathrm{~d}, \mathrm{~J}=7.6 \mathrm{~Hz}$, $0.5 \mathrm{H}, \mathrm{NHCO}), 5.25(\mathrm{~d}, \mathrm{~J}=8.4 \mathrm{~Hz}, 0.5 \mathrm{H}, \mathrm{NHCO}), 7.02(\mathrm{br} \mathrm{s}, 1 \mathrm{H}, \mathrm{NHBoc})$; HRMS $(\mathrm{M}+1)^{+}$; Calcd for $\mathrm{C}_{18} \mathrm{H}_{33} \mathrm{~N}_{2} \mathrm{O}_{5}: 357.23895$; Found: 357.23866 .

3-(Toluene-4-sulfonylamino)-4-hexyl-5-ethoxycarbonylpyrrolidin-2-one (27). The same procedure described for 26 was followed with diester $6(0.28 \mathrm{~g}, 0.58 \mathrm{mmol})$. Column chromatography (silica, hexane-EtOAc (1:1)) of the crude product gave $27 \mathrm{a}(0.05 \mathrm{~g}, 21 \%)$ and $27 \mathrm{~b}(0.05 \mathrm{~g}, 21 \%)$ as white solids; each solid was recrystallized from $\mathrm{CCl}_{4}$.

(27a) (more mobile): mp. 125-126 ${ }^{\circ} \mathrm{C}$; IR $\left(\mathrm{CCl}_{4}\right) 3300,2920,1730,1450,1335,1160 \mathrm{~cm}^{-1} ;{ }^{1} \mathrm{H}$ NMR $\left(\mathrm{CDCl}_{3}\right) \delta 0.90\left(\mathrm{~m}, 3 \mathrm{H}, \mathrm{CH}_{3}\left(\mathrm{CH}_{2}\right)_{5}\right), 1.2-1.4\left(\mathrm{~m}, 12 \mathrm{H}, \mathrm{OCH}_{2} \mathrm{CH}_{3}, \mathrm{CH}_{3}\left(\mathrm{CH}_{2}\right)_{4} \mathrm{CH}_{2}\right), 1.6-1.8(\mathrm{~m}, 1 \mathrm{H}$, $\left.\mathrm{CH}_{3}\left(\mathrm{CH}_{2}\right)_{4} \mathrm{CH}_{2}\right), 2.4\left(\mathrm{~m}, 1 \mathrm{H}, \mathrm{Hex}-\mathrm{CH}\right.$, and s, $\left.3 \mathrm{H}, \mathrm{C}_{6} \mathrm{H}_{4} \mathrm{CH}_{3}\right), 3.7(\mathrm{dd}, \mathrm{J}=7.6,7.6 \mathrm{~Hz}, 1 \mathrm{H}, \mathrm{CHNHTs}), 3.84$ $\left(\mathrm{d}, \mathrm{J}=7.0 \mathrm{~Hz}, 1 \mathrm{H}, \mathrm{CHCO}_{2} \mathrm{Et}\right), 4.22\left(\mathrm{q}, \mathrm{J}=7.0 \mathrm{~Hz}, 2 \mathrm{H}, \mathrm{OCH}_{2} \mathrm{CH}_{3}\right), 5.68(\mathrm{~d}, \mathrm{~J}=7.6 \mathrm{~Hz}, 1 \mathrm{H}, \mathrm{NHTs}), 6.78$ (s, $1 \mathrm{H}, \mathrm{NHCO}$ ), $7.30\left(\mathrm{~d}, \mathrm{~J}=8.0 \mathrm{~Hz}, 2 \mathrm{H}, 3,5-\mathrm{C}_{6} \mathrm{H}_{4} \mathrm{CH}_{3}\right), 7.81\left(\mathrm{~d}, \mathrm{~J}=8.0 \mathrm{~Hz}, 2 \mathrm{H}, 2,6-\mathrm{C}_{6} \mathrm{H}_{4} \mathrm{CH}_{3}\right) ; \mathrm{HRMS}$ $(\mathrm{M}+1)^{+}$; Calcd for $\mathrm{C}_{20} \mathrm{H}_{31} \mathrm{~N}_{2} \mathrm{O}_{5} \mathrm{~S}: 411.19537$; Found: 411.19525.

(27b) (less mobile): mp. $122-124{ }^{\circ} \mathrm{C}$; IR $\left(\mathrm{CCl}_{4}\right) 3200,1730,1700,1460,1335,1160 \mathrm{~cm}^{-1}$; ${ }^{1} \mathrm{H}$ NMR $\left(\mathrm{CDCl}_{3}\right) \delta 0.88\left(\mathrm{t}, \mathrm{J}=6.1 \mathrm{~Hz}, 3 \mathrm{H}, \mathrm{CH}_{3}\left(\mathrm{CH}_{2}\right)_{5}\right), 1.0-1.5\left(\mathrm{~m}, 12 \mathrm{H}, \mathrm{OCH}_{2} \mathrm{CH}_{3}, \mathrm{CH}_{3}\left(\mathrm{CH}_{2}\right)_{4} \mathrm{CH}_{2}\right), 1.6-1.8(\mathrm{~m}$, $\left.1 \mathrm{H}, \mathrm{CH}_{3}\left(\mathrm{CH}_{2}\right)_{4} \mathrm{CH}_{2}\right), 2.41\left(\mathrm{~m}, 1 \mathrm{H}, \mathrm{Hex}-\mathrm{CH}\right.$, and s, $\left.3 \mathrm{H}, \mathrm{C}_{6} \mathrm{H}_{4} \mathrm{CH}_{3}\right), 3.85\left(\mathrm{~d}, \mathrm{~J}=11 \mathrm{~Hz}, 1 \mathrm{H}, \mathrm{CHCO}_{2} \mathrm{Et}\right), 4.12$ 
(d, J = 7.2 Hz, $1 \mathrm{H}, \mathrm{CHNHTs}$ ), $4.20\left(\mathrm{q}, \mathrm{J}=6.9 \mathrm{~Hz}, 2 \mathrm{H}, \mathrm{OCH}_{2} \mathrm{CH}_{3}\right.$ ), 5.52 (br s, $1 \mathrm{H}, \mathrm{NHTs}$ ), 6.40 (br s, $1 \mathrm{H}$, $\mathrm{NHCO}), 7.30\left(\mathrm{~d}, \mathrm{~J}=8.3 \mathrm{~Hz}, 2 \mathrm{H}, 3,5-\mathrm{C}_{6} \mathrm{H}_{4} \mathrm{CH}_{3}\right), 7.80\left(\mathrm{~d}, \mathrm{~J}=8.3 \mathrm{~Hz}, 2 \mathrm{H}, 2,6-\mathrm{C}_{6} \mathrm{H}_{4} \mathrm{CH}_{3}\right)$; Anal. Calcd for $\mathrm{C}_{20} \mathrm{H}_{30} \mathrm{~N}_{2} \mathrm{O}_{5} \mathrm{~S}: \mathrm{C}, 58.51 ; \mathrm{H}, 7.37 ; \mathrm{N}, 6.82 ; \mathrm{S}, 7.81$; Found: C, 58.74; H, 7.33; N, 6.89; S, 7.66.

\section{REFERENCES}

1. For example, see: (a) Hruby, V. J. Trends Pharmacol. Sci. 1985, 6, 259. (b) Veber, D. F.; Freidinger, R. M.; Perlow, D. S.; Paleveda, W. J., Jr.; Holly, F. W.; Strachan, R. G.; Nutt, R. F.; Arison, B. H.; Homnick, C.; Randall, W. C.; Glitzer, M. S.; Saperstein, R.; Hirschmann, R. Nature 1981, $292,55$. (c) Freidinger, R. M.; Perlow, D. S.; Veber, D. F.; Brooks, J. R.; Saperstein, R. Science 1980, 210, 656. (d) Ward, P.; Ewan, G. B.; Jordan, C. C.; Ireland, S. J.; Hagan, R. M.; Brown, J. R. J. Med. Chem. 1990, 33, 1848. (e) Hinds, M. G.; Richards, N. G. J.; Robinson, J. A. J. Chem. Soc., Chem. Commun. 1988, 1447. (f) Aebi, J. D.; Guillaume, D.; Dunlap, B. E.; Rich, D. H. J. Med. Chem. 1988, 31, 1805. (g) Olson, G. L.; Voss, M. E.; Hill, D. E.; Kahn, M.; Madison, V. S.; Cook, C. M. J. Am. Chem. Soc. 1990, 112, 323. (h) Smith, A. B., III; Keenan, T. P.; Holcomb, R. C.; Sprengeler, P. A.; Guzman, M. C.; Wood, J. L.; Carroll, P. J.; Hirschmann, R. Ibid, 1992, 114, 10672. (i) Callahan, J. F.; Newlander, K. A.; Burgess, J. L.; Eggleston, D. S.; Nichols, A.; Wong, A.; Huffman, W. F. Tetrahedron 1993, 49. 3479. (j) Genin, M. J.; Gleason, W. B.; Johnson R. L. J. Org. Chem. 1993, 58, 860. (k) Baldwin, J. E.; Hulme, C.; Edwards, A. J.; Schofield, C. J. Tetrahedron Lett. 1993, 34, 1665. (l) Ripka, W. C.; De Lucca, G. V.; Bach, A. C., II; Pottorf, R. S.; Blaney, J. M. Tetrahedron 1993, 49, 3593. (m) Genin, M. J.; Ojala, W. H.; Gleason, W. B.; Johnson, R. L.; J. Org. Chem. 1993, 58, 2334. (n) Sreenivasan, U.; Mishra, R. K.; Johnson, R. L. J. Med. Chem. 1993, 36, 256. (o) Williams, B. J.; Curtis, N. R.; McKnight, A. T.; Maguire, J. J.; Young, S. C.; Veber, D. F.; Baker, R. Ibid, 1993, 36, 2. (p) Robl, J. A.; Cimarusti, M. P.; Simpkins, L. M.; Weller, H. N.; Pan, Y. Y.; Malley, M.; DiMarco, J. D. J. Am. Chem. Soc. 1994, 116, 2348. (q) Gante, J. Angew. Chemie 1994, 106, 1780.

2. (a) Freidinger, R. M.; Perlow, D. S.; Veber, D. F. J. Org. Chem. 1982, 47, 104. (b) Garvey, D. S.; May, P. D.; Nadzan, A. M. Ibid, 1990, 55, 936.

3. For synthesis of 3-amino-3-substituted-pyrrolidin-2-ones, see: (a) Freidinger, R. M. J. Org. Chem. 1985, 50, 3631. (b) Holladay, M. W.; Nadzan, A. M. Ibid, 1991, 56, 3900. (c) Zydowsky, T. M.; Dellaria, Jr., J. F.; Nellans, H. N. Ibid, 1988, 53, 5607. (d) Thaisrivongs, S.; Pals, D. T.; Turner, S. R.; Kroll, L. T. J. Med. Chem. 1988, 31, 1369. (e) Abell, A. D.; Taylor, J. M. J. Org. Chem. 1993, 58, 14.

4. (a) Legters, J.; Thijs, L.; Zwanenburg, B. Tetrahedron Lett. 1989, 30, 4881. Legters, J.; Thijs, L.; Zwanenburg, B. Recl. Trav. Chim. Pays-Bas 1992, 111, 1. (b) Legters, J. Ph.D. Thesis; 1991. University of Nijmegen.

5. Gao, Y.; Hanson, R. M.; Klunder, J. M.; Ko, S. Y.; Masamune, H.; Sharpless, K. B. J. Am. Chem. Soc. $1987,109,5765$.

6. Bergmeier, S. C.; Lee, W. K.; Rapoport, H. J. Org. Chem., 1993, 58, 5019.

7. Fitremann, J.; Duréault, A.; Depezay, J-C. Tetrahedron Lett., 1994, 35, 1201.

8. Aza-[2,3]-Wittig rearrangement of vinylaziridines, see: Åhman, J.; Somfai, P. J. Am. Chem. Soc., 1994, 116, 9781; Ånman, J.; Somfai, P. Tetrahedron Lett. 1995, 36, 303; Coldham, I.; Collis, A. J.; Mould, R. J.; Rathmell, R. E. Ibid, 1995, 36, 3557.

9. (a) Deyrup, J. A. in Heterocyclic Compounds, vol. 42, part 1, "Small Ring Heterocycles", pp. 115-119, Hassner, A. ed., Wiley Interscience, New York, 1983. (b) Bouayad, Z.; Chanet-Ray, J.; Ducher, S.; Vessiére, R. J. Heterocyclic Chem. 1991, 28, 1757. (c) Stamm, H.; Schneider, L. Chem. Ber. 1975, 108, 500. (d) Hassner, A.; Kascheres, A. Tetrahedron Lett. 1970, 4623. (e) Baldwin, J. E.; Adlington, R. M.; O'Neil, I. A.; Schofield, C.; Spivey, A. C.; Sweeney, J. B. J. Chem. Soc., Chem. Commun. 1989, 1852. 
10. (a) Nakajima, K.; Neya, M.; Yamada, S.; Okawa, K. Bull. Chem. Soc. Jpn. 1982, 55, 3049. (b) Baldwin, J. B.; Adlington, R. M.; Robinson, N. G. J. Chem. Soc., Chem. Commun. 1987, 153. (c) Sato, K.; Kozikowsky, A. P. Tetrahedron Lett. 1989, 30, 4073. (d) Shima, I.; Shimazaki, N.; Imai, K.; Hemmi, K.; Hashimoto, M. Chem. Pharm. Bull. 1990, 38, 564. (e) Okawa, K.; Nakajima, K.; Tanaka, T.; Neya, M. Bull. Chem. Soc.Jpn. 1982, 55, 174. (f) Nakajima, K.; Tanaka, T.; Morita, K.; Okawa, K. Ibid, 1980, 53, 283. (g) Parry, R. J.; Naidu, M. V. Tetrahedron Lett. 1983, 24, 1133. (h) Vaultier, M.; Ouali, M. S.; Carrié, R. Bull. Soc. Chim. Fr. Part II, 1979, 343.

11. The X-ray data will be submitted for publication separately: Smits, J.; Beurskens, P. T.; Israel, O. R.; Funaki, I.; Thijs, L.; Zwanenburg, B. Acta Crystallographica Sec. C.

12. Ho, T-L. Chem. Rev. 1975, 75, 1.

13. (a) Intramolecular attack at the C-2 position in aziridinecarboxamides will be published in due time. For intramolecular ring-opening reactions of epoxycarboxamides, see: (b) Shiozaki, M.; Ishida, N.; Hiraoka, T.; Yanagisawa, H. Tetrahedron Lett. 1981, 22, 5205. (c) Yanagisawa, H.; Ando, A.; Shiozaki, M.; Hiraoka, T. Ibid, 1983, 24, 1037. (d) Chackalamannil, S.; Fett, N.; Kirkup, M.; Afonso, A.; Ganguly, A. K. J. Org. Chem. 1988, 53, 450. (e) Hanessian, S; Bedeschi, A.; Battistini, C.; Mongelli, N. J. Am. Chem. Soc. 1985, 107, 1438. (f) Häbich, D.; Hartwig, W. Tetrahedron Lett. 1987, 28, 781.

14. For full details, see: Funaki, I. Thesis University of Nijmegen, 1996. 2D-NOESY NMR study application to 1,3-imidazolidin-5-ones, see: Grozinger, K. G.; Kriwacki, R. W.; Leonard, S. F.; Pitner, T. P. J. Org. Chem. 1993, 58, 709.

15. Cruickshank, P. A.; Fishman, M. J. Org. Chem. 1969, 34, 4060.

16. Electronic effects of substituents on intramolecular epoxide ring-opening reactions, see: Nicolaou, K. C.; Prasad, C. V. C.; Somers, P. K.; Hwang, C.-K. J. Am. Chem. Soc. 1989, 111, 5330.

17. Krapcho, A. P.; Lovey, A. J. Tetrahedron Lett. 1973, 957.

18. Kanemasa, S.; Tatsukawa, A.; Wada, E. J. Org. Chem. 1991, 56, 2875.

19. Von Arx, E.; Faupel, M.; Brugger, M. J. J. Chromatogr. 1976, I20, 224.

20. Org. Synth., Collective Vol., V, pp. 373-378.

21. Luo, F.-T.; Negishi, E.-I. J. Org. Chem. 1985, 50, 4762.

(Received in UK 29 April 1996; revised 28 May 1996; accepted 30 May 1996) 